Publ. RIMS, Kyoto Univ.

12 Suppl. (1977), 387-438.

\title{
Holonomy Structure of Landau Singularities and Feynman Integrals
}

\author{
By \\ Mikio SATo*, Tetsuji MiwA*, Michio Jimbo* and Toshio Oshima**
}

\section{Introduction}

Since the pioneering work of Landau and Nakanishi on general Feynman integrals, it has been known that the singularities of the $S$-matrix, causal Green's functions and related functions are described by the so called Landau equations. These Landau singularities were physically interpreted as the macroscopic causality by the theoretical physicists working in $S$-matrix theory, and the notion of essential support was obtained (Chandler-Stapp [3], Iagolnitzer-Stapp [5]).

In the branch of mathematics, on the other side, the theory of microfunction has evolved and has been applied powerfully to the general theory of partial differential equations (Sato-Kawai-Kashiwara [11]). It contained the essential support theory as the singularity spectrum of a function (i.e. its support viewed as a microfunction), and was far-reaching because of its close connection with the theory of differential equations. Namely the method of microlocal analysis, based on the theory of holonomic systems, has provided a systematic way of handling functions with "natural background" and found most effective applications to various problems of mathematics, such as the theory of $b$-functions and Fourier transformations (Sato [10], Kashiwara [6]). It was then recognized that the Landau equations give holonomic varieties, which led one to the holonomicity postulate of $S$-matrix and related quantities (Sato [10]). In the present paper we shall study the holonomy structure of Landau singularities and Feynman integrals from this standpoint.

First we review the notion of Landau varieties. In contrast with

Received October 12, 1976.

* Research Institute for Mathematical Sciences, Kyoto University, Kyoto 606, Japan.

** Department of Mathematics, Faculty of Science, The University of Tokyo, Tokyo 113, Japan. 
the usual presentation, they are defined as subvarieties of the phase space $T^{*} C^{\nu n}$. The importance of this point of view will be clarified in later paragraphs. In $\S 2$ we study the holonomy structure assuming that the Feynman graph in question has only external vertices. An explicit formula is obtained for calculating the dimension of a Landau variety considered in the momentum space. From the general consideration on this formula we are led to the notion of pseudo-graphs (§3). For the most general Feynman graphs, the situation is more involved. The Landau variety is no longer irreducible nor manifestly holonomic in general. Regarding the mass parameters as variables, we show the existence and the holonomicity of a unique irreducible component characterized by the condition $U(\alpha) \not \equiv 0$. In $\S 5$ we try to classify all the irreducible components of a vacuum graph (a graph without external vertices) which have 2dimensional realizations. $\S 6$ and $\S 7$ constitute the analytical part of this paper and are devoted to the study of Feynman integrals. A rigorous definition, shown to be an equivalent of Speer's ([13]), is given through a compactification of the momentum space. As a result the following well known property is established for a most general Feynman integral: its singularity spectrum is confined to the positive- $\alpha$ Landau varieties (cf. Chandler [2]). In the case of an external graph, the singularity structure is determined at a 1-codimensional intersection of two Landau varieties, where a microlocal version of the local decomposition theorem of Speer-Westwater [15] is obtained by the aid of the quantized contact transformation. An application to the generalized unitarity relations is given in $\S 7$.

\section{§ 1. Landau Equations}

In this section we recall briefly the definitions of Landau equations and Landau holonomic varieties.

Imagine a classical collision process of elementary particles in 4dimensional space-time.

In Figure (1.1), two particles with 4 -momenta $-p_{1}$ and $-p_{2}$ collide at $x_{1}$ and produce $k_{1}$ and $k_{2}$, then $-p_{3}$ collides with $k_{2}$ at $x_{2}$ and so forth.

${ }^{\dagger}$ An orientation $\omega$ is a collection of numbers $\omega_{l}= \pm 1$ assigned to each internal line $l \in I_{0}$. 




Fig. (1.1)

Physical conservation law requires the following set of equations called the Landau equations for the diagram of Figure (1.1):

(I) (energy-momentum conservation)

$$
-p_{1}-p_{2}=k_{1}+k_{2}, \quad-p_{3}-p_{6}=-k_{2}+k_{3}, \quad p_{4}+p_{5}=k_{1}+k_{3},
$$

(II) (circuit condition) there exist positive numbers $\alpha_{l}(l=1,2,3)$ such that

$$
x_{3}-x_{1}=\alpha_{1} k_{1}, \quad x_{2}-x_{1}=\alpha_{2} k_{2}, \quad x_{3}-x_{2}=\alpha_{3} k_{3},
$$

(III) (mass shell constraint)

$$
k_{l}{ }^{2}=m_{l}{ }^{2} \quad \text { for } \quad l=1,2,3 .
$$

Here $k_{l}{ }^{2}=k_{l 0}^{2}-k_{l 1}^{2}-k_{l 2}^{2}-k_{l 3}^{2}$ is the Lorentz metric, and the $m_{l}$ 's are the rest masses of the corresponding intermediate particles. ${ }^{(\dagger)}$

In general, a Feynman graph is a triple $\left(G, J_{0}, \sigma\right)$ consisting of (i) a linear graph $G$ (either oriented ${ }^{(\dagger+)}$ or non oriented), (ii) a set of indices $J_{0}=\left\{1, \cdots, n_{0}\right\}$, called the set of external momenta, and (iii) a mapping $\sigma: J_{0} \rightarrow\{1, \cdots, n(G)\}$ to the set of vertices of $G$. A vertex $j$ of $G$ is called external if $\sigma^{-1}(j) \neq \phi$. Otherwise it is called an internal vertex.

We shall use the following notations.

$$
\begin{aligned}
& n(G)=\text { the number of vertices of } G \\
& N(G)=\text { the number of internal lines of } G \\
& b_{0}(G)=\text { the number of connected components of } G \\
& b_{1}(G)=\text { the number of independent loops of } G \\
& \chi(G)=b_{0}(G)-b_{1}(G)=n(G)-N(G)
\end{aligned}
$$

"We work in the "off-mass-shell" frame, that is we do not require the mass shell constraint for external momenta $p_{j}$ 's. 


$$
\rho(G)=N(G)-b_{1}(G)=n(G)-b_{0}(G)
$$

Consider the momentum space $\boldsymbol{R}^{\nu}$ equipped with the Lorentz metric $p^{2}={p_{0}}^{2}-\sum_{\mu=1}^{\nu-1} p_{\mu}{ }^{2}$. We identify the dual space (the configuration space) $\left(\boldsymbol{R}^{\nu}\right)^{*}$ with $\boldsymbol{R}^{\nu}$ by this metric. For a Feynman graph $(G, \sigma)^{\dagger}$, Landau holonomic variety is defined in the cotangent bundle $T^{*} \boldsymbol{R}^{\nu n_{0}}$ as follows: (the case $\sigma=i d$ ).

The positive- $\alpha$ Landau holonomic variety is defined by

$$
\Lambda_{G}{ }^{+}=\text {closure of }\left\{(p ; x) \in T^{*} \boldsymbol{R}^{\nu n} \mid \exists \alpha_{l}>0, \quad \exists k_{l} \in \boldsymbol{R}^{\nu}(l=1, \cdots, N)\right.
$$

such that

$$
\begin{array}{ll}
p_{j}=\xi_{j}(k) \equiv \sum_{l=1}^{N}[l: j] k_{l} & (j=1, \cdots, n) \\
\alpha_{l} k_{l}=\eta_{l}(x) \equiv \sum_{j=1}^{n}[l: j] x_{j} & (l=1, \cdots, N) \\
k_{l}{ }^{2}=m_{l}{ }^{2} & (l=1, \cdots, N)\},
\end{array}
$$

where $[l: j]$ denotes the incidence number, and the $m_{l}$ 's are given non negative constants. ${ }^{(\dagger)}$ Equations (I), (II), (III) are called the Landau equations for the graph $G$. In the complexification $T^{*} \mathbb{C}^{\nu n}$ we define the Landau holonomic veriety by

$$
\begin{gathered}
\Lambda_{G}{ }^{C}=\text { closure of }\left\{(p ; x) \in T^{*} C^{\nu n} \mid \exists \alpha_{l} \in C-\{0\}, \quad \exists k_{l} \in C^{\nu}(l=1, \cdots, N)\right. \\
\text { such that (I), (II), (III) hold }\} .
\end{gathered}
$$

(general case)

For a general graph $(G, \sigma)$ we set $q_{j}(p)=\sum_{i \in \sigma^{-1}(j)} p_{i}$ for each vertex $j$ of $G$, which reduces to 0 if $\sigma^{-1}(j)=\phi$. Then the positive- $\alpha$ Landau holonomic variety for $(G, \sigma)$ is defined by

$$
\begin{aligned}
& \Lambda_{G, \sigma}^{+}=\left\{(p ; x) \in T^{*} \boldsymbol{R}^{\nu n_{0}} \mid \exists y_{j} \in \boldsymbol{R}^{\nu} \quad(j=1, \cdots, n(G)),\right. \\
& \text { such that } \left.(q(p) ; y) \in \Lambda_{G}^{+} \text {and } x_{i}=y_{\sigma(i)}\left(i=1, \cdots, n_{0}\right)\right\} .
\end{aligned}
$$

We call its projection to the base space $L_{G, \sigma}^{+}=\overline{\pi\left(\Lambda_{G, \sigma}^{+}\right)}\left(\pi: T^{*} \boldsymbol{R}^{\nu n_{0}} \rightarrow \boldsymbol{R}^{\nu n_{0}}\right)$ the positive- $\alpha$ Landau singularity. $\Lambda_{G, \sigma}^{\boldsymbol{C}}$ and $L_{G, \sigma}^{\boldsymbol{C}}$ are defined in a similar manner.

Note that these definitions do not depend on the choice of a particular

\footnotetext{
t In that follows we denote a Feynman graph simply by $(G, \sigma)$ or $G$.

${ }^{\dagger}$ In $\S 2, \S 3$ and $\S 4$ we assume that $G$ is massive, that is, $m_{l}>0$ for any $l$.
} 
orientation of $G$. For instance, $\Lambda_{G}{ }^{+}$is represented as $\Lambda_{G}{ }^{+}=\overline{\bigcup_{\omega} \Lambda_{G}{ }^{\omega}}$, where

$$
\begin{aligned}
\Lambda_{G}{ }^{\omega}= & \left\{\left.(p ; x) \in T^{*} \boldsymbol{R}^{\nu n}\right|_{\mid} ^{\exists} \alpha_{l}>0,{ }^{\exists} k_{l} \in \boldsymbol{R}^{\nu} \text { with } k_{l 0}>0\right. \\
& \text { such that (I), (II), (III) hold for the orientation } \omega\},
\end{aligned}
$$

and the union is taken over all the possible orientations of $G$.

Denote by $G^{(i)}(i=1, \cdots, r)$ the connected components of $G$, and define the space of overall conservation by

$$
P_{0}(G)=\left\{p \in C^{n n_{0}} \mid \sum_{\sigma(j) \in \text { vertices of } G(i)} p_{j}=0(i=1, \cdots, r)\right\} .
$$

From the energy-momentum conservation (I), it follows that $L_{G, \sigma}^{C} \subset P_{0}(G)$ and $\Lambda_{G, \sigma}^{\boldsymbol{C}}$ may be regarded as a subvariety of $T^{*} P_{0}$. In this context we sometimes call $P_{0}(G)$ or its conormal bundle $T_{P_{0}(G)}^{*} C^{\nu n_{0}}$ the zero-section.

Let $(G, \sigma)$ be a Feynman graph, $I$ be a subset of internal lines and $G / I$ the graph obtained by contracting lines in $I$ of $G$. Then we have a natural mapping of vertices $\tau_{I}:\{1, \cdots, n(G)\} \rightarrow\{1, \cdots, n(G / I)\}$. The (non-leading) Landau holonomic variety corresponding to the contraction $G / I$ is defined by $\Lambda_{G / I, \tau_{I} \sigma}^{\boldsymbol{C}}$.

\section{§ 2. Holonomy Structure (External Case)}

In this section we determine the holonomy structure of Landau singularities of an external graph using a contact transformation and the codimension formula.

We consider external graphs-graphs with no internal vertices. ${ }^{(\dagger)}$ In this case we can eliminate the $k_{l}$ 's and $\alpha_{l}$ 's from the Landau equation (I), (II), (III) to obtain a closed expression for $\Lambda_{G}{ }^{\mathbb{C}}$ :

$$
\begin{gathered}
\Lambda_{G}{ }^{c}=\text { closure of }\left\{(p ; x) \in T^{*} C^{\nu n} \mid p_{j}=\frac{\partial H_{G}}{\partial x_{j}}(j=1, \cdots, n),\right. \\
\left.\eta_{l}(x)^{2} \neq 0 \quad(l=1, \cdots, N)\right\} .
\end{gathered}
$$

Here $H_{G}(x)=\sum_{l=1}^{N} m_{l}{\sqrt{\eta_{l}(x)^{2}}}^{2}$, the potential function as we call it, is a multi-valued analytic function, homogeneous of degree 1 . If we denote

\footnotetext{
${ }^{\dagger}$ So we may assume $\sigma=i d$ without loss of generality.
} 
the fundamental 1 -form by $\omega=\sum_{j=1}^{n} x_{j} d p_{j}$, we have

$$
\begin{aligned}
\left.\omega\right|_{A_{G}} c & =d\left(\sum_{j=1}^{n} x_{j} p_{j}\right)-\left.\sum_{j=1}^{n} p_{j} d x_{j}\right|_{\Lambda_{G}} c \\
& =d\left(\sum_{j=1}^{n} x_{j} \frac{\partial H}{\partial x_{j}}\right)-\sum_{j=1}^{n} \frac{\partial H}{\partial x_{j}} d x_{j} \\
& =d H-d H=0,
\end{aligned}
$$

so $\Lambda_{G}{ }^{c}$ is in fact a holonomic variety. By the aid of the potential function, we can define the following contact transformation. Let $I_{0}=\{1, \cdots, N\}$ be the set of internal lines of $G$ and $I$ be its subset. We denote by $G_{I}$ the graph obtained by deleting lines in $I_{0}-I$ from $G$. Divide the potential function into two parts $H_{G}(x)=H_{G_{I_{0}-I}}(x)+H_{G_{I}}(x)$. Then the mapping $(p ; x) \rightarrow(\tilde{p} ; \tilde{x})$ with

$$
\widetilde{p}_{j}=p_{j}-\frac{\partial}{\partial x_{j}} H_{G_{I_{0}-I}}, \widetilde{x}_{j}=x_{j}
$$

is well defined if $\eta_{l}(x)^{2} \neq 0$ for all $l \in I_{0}-I$, and the homogeneity of $H_{G_{I_{0}-I}}$ shows that this is in fact a contact transformation. In terms of the coordinate $(\widetilde{p} ; \widetilde{x})$, the defining equations for $\Lambda_{G}{ }^{c}$ reads

$$
\widetilde{p}_{j}=\frac{\partial}{\partial \widetilde{x}_{j}} H_{G_{I}}(\widetilde{x}) \quad(j=1, \cdots, n),
$$

which are nothing but the defining equations for $\Lambda_{G_{I}}^{C}$. Moreover, $(2 \cdot 1)$ is also defined on $\Lambda_{G / I^{\prime}, \tau_{I^{\prime}}}^{\boldsymbol{C}}$ for any $I^{\prime} \subset I$, and transforms it onto $\Lambda_{G_{I} / I^{\prime}}^{\boldsymbol{C}}, \tau_{I^{\prime}}$. These correspondences are illustrated as follows:

$$
\begin{aligned}
& \Lambda_{G}^{C} \leftrightarrow \Lambda_{G}^{C} \\
& \Lambda_{G / I^{\prime}, \tau_{I}}^{C} \leftrightarrow \Lambda_{G_{I} / I^{\prime}, \tau_{I^{\prime}}}^{C}\left(I^{\prime} \subset I\right)
\end{aligned}
$$



Fig. (2.1) 
The graph $G_{I} / I$ consists of only isolated points, and $\Lambda_{G_{I} / I, \tau_{I}}^{C}$ is the "zero section", i.e. the space of overall energy-momentum conservation:

$$
\begin{aligned}
\Lambda_{G_{I} / I, \tau_{I}}^{G}= & \left\{(p ; x) \mid \sum_{\text {vertices of } G_{I}(i)} p_{j}=0 \text { and } x_{j}=x_{j^{\prime}}\right. \\
& \text { for any vertices } \left.j, j^{\prime} \text { of } G_{I}^{(i)}\right\}
\end{aligned}
$$

where $G_{I}^{(i)}(i=1, \cdots, r)$ denote the connected components of $G_{I}$. Hence $\Lambda_{G}^{\boldsymbol{C}} \cap \Lambda_{G / I, \tau_{I}}^{\boldsymbol{C}}$ is transformed onto $\Lambda_{G_{I}}^{\boldsymbol{C}} \cap \Lambda_{G_{I} / I, \tau_{I}}^{\boldsymbol{C}}=\left\{(p ; x) \in T^{*} \mathbb{C}^{\nu n} \mid p \in L_{G_{I}}^{\boldsymbol{C}}, x_{j}\right.$ $=x_{j}$, for any vertices $j, j^{\prime}$ of $\left.G_{I}^{(i)}\right\}$.

Theorem 2.1. $\Lambda_{G}^{C}$ and $\Lambda_{G / I, \tau_{I}}^{C}$ intersects regularly, and codim $\Lambda_{G}^{C} \cap \Lambda_{G / I, \tau_{I}}^{C}$ in $\Lambda_{G}^{C}=\operatorname{codim} L_{G_{I}}^{C}$ in $\mathbb{C}^{\nu n}-\nu b_{0}\left(G_{I}\right)$. By "regular intersection" we mean that

(i) $\Lambda_{G}^{C}$ and $\Lambda_{G / I, \tau_{I}}^{C}$ are non singular at a generic point $P_{0}{ }^{*}$ of $\Lambda_{G}^{C} \cap \Lambda_{G / I, \tau_{I}}^{C}$, and



Proof. As we have remarked in $\S 1, L_{G_{I}}^{\mathbb{C}} \subset P_{0}\left(G_{I}\right)$, and $\operatorname{codim} P_{0}\left(G_{I}\right)$ in $\mathbb{C}^{\nu n}$ is equal to $\nu b_{0}\left(G_{1}\right)$, hence the theorem follows immediately.

The following formula tells us how to calculate the codimension of $L_{G}{ }^{C}$. The proof is given in $\S 3$.

Theorem 2.2. (codimension formula)

$$
\operatorname{codim} L_{G}{ }^{C}=\nu \chi(G)+\max _{I_{0}=I_{1} \sqcup \ldots \cup_{I_{k}}} \sum_{k=1}^{k}\left(1+\nu b_{1}\left(G_{I_{k}}\right)\right)
$$

where $I_{1} \sqcup \cdots \sqcup I_{k}$ runs over all partitions of the set $I_{0}$.

It is easy to rewrite the above formula to the dimension formula.

Theorem 2.3. (Dimension formula)

$$
\operatorname{dim} L_{G} \boldsymbol{C}^{\boldsymbol{C}}=\min _{\boldsymbol{I}_{0}=I 1^{\cup} \ldots \cup_{\boldsymbol{I}_{k}}} \sum_{k=1}^{k}\left(-1+\nu \rho\left(G_{I_{k}}\right)\right)
$$


A partition will be called good if it attains the maximum (or the minimum) in the formulas. When the trivial partition $I_{0}$ is good, we say that $G$ is a primary graph. Note that this depends on the dimension $\nu$ of space-time. Since the union of two primary graphs with an internal line in common is itself primary (see $\S 3$ ), there exists a unique partition of $I_{0}$ composed of all the maximal primary subgraphs of $G$.

Proposition 2.4. A maximal primary partition is good.

Proof. Any good partition consists of primary subgraphs, hence it is a refinement of a maximal primary partition. Then it is easy to see that the latter is also a good partition.

Example 2.5. (1-loop graph) $\operatorname{codim} L_{G} \boldsymbol{c}=\max (\nu+1, N)$, and $G$ is primary if and only if $\nu+1 \geqq N$.

Example 2.6. (2-loop graph) $\operatorname{codim} L_{G} \boldsymbol{C}=\max \left(\nu+1, N_{1}+1, N_{2}+1, N_{3}+1, N_{1}+N_{2}+N_{3}-\nu\right)$

where $N_{i}$ is the number of internal lines composing each arm (Fig. 2.2).

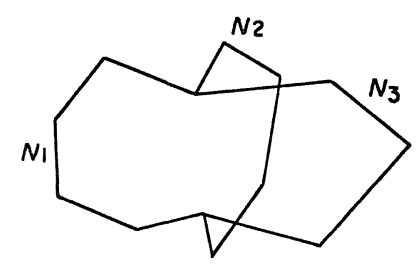

Fig. (2.2)

Proposition 2.7. codim $L_{G} \boldsymbol{c}=\nu b_{0}(G)+1$ if and only if $G$ is primary.

Theorem 2.8. $\Lambda_{G}^{C}$ and $\Lambda_{G / I, \tau_{I}}^{C}$ have 1-codimensional intersection if and only if $G_{I}$ is primary.

By this theorem the holonomy diagram for a Feynman integral is completely determined in the case of external graphs, except for the 
second type singularities which we do not discuss here.

Holonomy diagram for a 2-loop graph $(\nu=2)$


Fig. (2.3)

When $\Lambda_{G}{ }^{C}$ and $\Lambda_{G / I, \tau_{I}}^{C}$ have 1-codimensional intersection, the relation between $\operatorname{codim} L_{G}^{\boldsymbol{c}}$ and $\operatorname{codim} L_{G / I, \tau_{I}}^{\boldsymbol{C}}=\operatorname{codim} L_{G / I}^{\boldsymbol{C}}$ is given by the following theorem. The proof is given in $\S 3$.

Theorem 2.9. Let $G_{0}$ be the maximal primary subgraph containing $G_{I}$. Then

$$
\begin{aligned}
\operatorname{codim} L_{G} \boldsymbol{C}-\operatorname{codim} L_{G / I}^{\boldsymbol{C}} & =\operatorname{codim} L_{G_{0}}^{\boldsymbol{C}}-\operatorname{codim} L_{G_{0} / I}^{\boldsymbol{C}} \\
& =-1,0,1,2, \cdots
\end{aligned}
$$

In particular,

$\operatorname{codim} L_{G}^{\boldsymbol{C}}-\operatorname{codim} L_{G / I}^{\boldsymbol{C}}=-1$ if and only if $G_{I}$ is maximal primary, $\operatorname{codim} L_{G}{ }^{\boldsymbol{C}}-\operatorname{codim} L_{G / I}^{\boldsymbol{C}}=0$ if and only if $G_{0} / I$ is primary.

\section{$\S 3$. The proof of codimension formula}

In this section we prove the codimension formula (Theorem 2.2) and give its several applications.

In proving the codimension formula, we also consider a real locus $\Lambda_{G}{ }^{\omega}$ for a fixed orientation $\omega$. On $\Lambda_{G}^{\omega}$, the $k_{l}$ 's are uniquely determined by $p$. For if $p_{j}=\sum_{l}[l: j] k_{l}=\sum_{l}[l: j] \tilde{k}_{l}$ for any $j$,

$$
0=\sum_{j} x_{j}\left(\sum_{l}[l: j] k_{l}-\sum_{l}[l: j] \tilde{k}_{l}\right)=\sum_{l} \alpha_{l}\left(k_{l}{ }^{2}-k_{l} \cdot \tilde{k}_{l}\right)
$$

where $\alpha_{l}>0$, and $k_{l}, \quad \tilde{k}_{l}$ are positive timelike $\nu$-vectors with the same length $m_{l}$. Applying Schwarz' inequality, we get $k_{l}=\tilde{k}_{l}$. Moreover the following holds: 
Proposition 3.1. (cf. Chandler-Stapp [3]) $k_{l}=k_{l}(p)$ can be extended as a continuous function on the closure $\overline{L_{G}}{ }^{\omega}$.

\section{Lemma 3.2. $\overline{L_{G}{ }^{\omega}} \subset \underset{I \subsetneq I_{0}}{\cup} L_{G / I, \tau_{I}}^{\omega}$.}

This lemma is proved by the same argument as in the page 853 of Chandler-Stapp [2] where the case when $G$ is primary is treated. So we omit its proof.

Proof of Proposition 3.1. By the above argument $k_{l}(p)$ is continuous on $L_{G}{ }^{\omega}$. Let $p^{0} \in \overline{L_{G}{ }^{\omega}}$, then by Lemma 3.2. we can find $I \subsetneq I_{0}$ such that $p_{0} \in L_{G / I, \tau_{1}}^{\omega}$. Hence if $l \in I_{0}-I, k_{l}(p)$ is also well-defined and continuous. For $l \in I$, by an induction we may assume that there is a continuous extension $k_{l}^{{ }^{G}}(p)$ on $\overline{L_{G_{I}}^{\omega}}$. If we put

$$
p_{I j}(p)=p_{j}-\sum_{l \in I_{0}-I}[l: j] k_{l}(p) \quad(j=1, \cdots, n)
$$

then $p \in L_{G}^{\omega}$ implies $p_{I} \in L_{G_{I}}^{\omega}$, hence $p_{I}\left(p^{0}\right) \in \overline{L_{G_{I}}^{\omega}}$. Thus we can define $k_{l}\left(p^{0}\right)=k_{l}^{G_{I}}\left(p_{I}\left(p^{0}\right)\right)(l \in I)$ and obtain a continuous extension.

Denote by $\pi$ the projection $T^{*} \mathbb{R}^{\nu n} \rightarrow \mathbb{R}^{\nu n}$, and its restriction to $\Lambda_{G}{ }^{\omega}$ by $\pi_{\omega}$. The above argument shows

$(3 \cdot 1) \quad \pi_{\omega}^{-1}(p)=\left\{(p ; x) \in T^{*} \mathbb{R}^{\nu n} \mid \sum_{j=1}^{n}[l: j] x_{j}=\alpha_{l} k_{l}(p)\right.$ for some $\left.\alpha_{l}>0\right\}$.

Since $\Lambda_{G}{ }^{\omega}$ is holonomic, $\operatorname{dim} \Lambda_{G}{ }^{\omega}=\nu n$ and

$$
\operatorname{codim} L_{G}{ }^{\omega}=\nu n-\operatorname{rank} \pi_{\omega}=\min _{p \in L_{G}} \operatorname{dim} \pi_{\omega}^{-1}(p) .
$$

From $(3 \cdot 1)$ we see that $\operatorname{dim} \pi_{\omega}^{-1}(p)$ is the dimension of mobility of the graph under fixed $k_{l}$ 's. Furthermore this coincides with the corank of $d \pi_{\omega}$ on $\pi_{\omega}^{-1}(p)$, which indicates the

Proposition 3.3. Let $L_{0}$ be the set of points in $L_{G}^{\omega}$ where $\operatorname{dim} \pi_{\omega}^{-1}(p)$ takes its minimum. Then

$$
L_{G}{ }^{\omega}-L_{0}=\text { the set of singular points of } L_{G}{ }^{\omega} \text {. }
$$

Thus both the problems of determining

(i) $\operatorname{codim} L_{G}$, 
and (ii) singularities of $L_{G}{ }^{\omega}$

are reduced to calculating this mobility of the graph in a given configuration.

For example, take a triangle graph $G$ with $\nu \geqq 2$.



general configuration

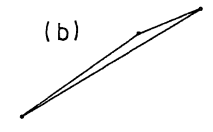

singular configuration

Fig. (3.1)

In its general configuration, the graph has no mobility, apart from translation and similar enlargement, without changing the direction of lines, so $\operatorname{codim} L_{G}=\nu+1$ (that is, $G$ is a primary graph). But when all the $k_{l}$ ' $s$ are parallel (Fig. 3.1 (b)), the mobility becomes $\nu+2$ and the corresponding $p$ is a singular point of $L_{G}{ }^{\omega}$.

In order to calculate the mobility of a graph, we prove a theorem of linear algebra. Before explaining it, it is useful to generalize the notion of a graph and introduce a pseudo graph.

Definition 3.4. We call $G=\left(U \stackrel{\eta_{l}}{\rightarrow} V_{l} \mid l \in I_{0}\right)$ a pseudo graph if $U$ and $V_{l}$ 's are vector spaces over a field $K$ and $\eta_{l}$ 's are non trivial linear mappings. For a subset $I \subset I_{0}$, we can construct two pseudo graphs;

and

$$
\text { restriction } G_{I}=\left(U \stackrel{\eta_{l}}{\rightarrow} V_{l} ! l \in I\right)
$$

where

$$
\text { contraction } G / I=\left(U_{G / I} \stackrel{\eta_{l}}{\rightarrow} V_{l} \mid l \in I_{0}-I\right)
$$

$$
U_{G / I}=\left\{x \in U \mid \eta_{l}(x)=0 \text { for } l \in I\right\} \text {. }
$$

We only consider such a contraction that $\left.\eta_{l}\right|_{U_{G / I}} \neq 0$ for any $l \in I_{0}-I$. We have the following exact sequence

$$
U^{t C} \rightarrow V=\underset{l \in I_{0}}{\oplus} V_{l} \stackrel{D}{\rightarrow} W \rightarrow 0
$$

where $\left({ }^{t} C x\right)_{l}=\eta_{l}(x)$. If $G$ is a graph and $C=([j: l])$ is its incidence matrix, taking $U=K^{\nu n(G)}, V_{l}=K^{\nu}$ and $\eta_{l}(x)=\sum_{j=1}^{n}[l: j] x_{j}$, we obtain a pseudo graph. In this context we use the following notations.

$$
\rho(G)=\operatorname{rank} C, \quad b_{0}(G)=\operatorname{dim} \operatorname{Ker}^{t} C, \quad b_{1}(G)=\operatorname{rank} D .
$$

To express the mobility of a pseudo graph we consider 


$$
\begin{aligned}
& U_{x_{0}}(G)=\left\{x \in U \mid \eta_{l}(x) / / \eta_{l}\left(x_{0}\right) \text { for any } l \in I_{0}\right\}, \\
& \operatorname{dim}_{G, x_{0}} I=\operatorname{rank}\left(U_{x_{0}}(G) \stackrel{t^{t C}}{\rightarrow} V_{I}\right),
\end{aligned}
$$

where $V_{I}=\bigoplus_{l \in I} V_{l}$ and $\operatorname{dim}_{G} I=\min _{x_{0} \in U} \operatorname{dim}_{G, x_{0}} I$.

We also introduce Landau singularity for a pseudo graph:

$\mathcal{L}_{G}{ }^{\boldsymbol{c}}=$ Zariski closure of

$$
\left\{\left[\eta_{l}(x)\right]_{l \in I_{0}} \in \prod_{l \in I_{0}} \boldsymbol{P}\left(V_{l}\right) \mid x \in U, \eta_{l}(x) \neq 0 \text { for any } l \in I_{0}\right\}
$$

where $\boldsymbol{P}\left(V_{l}\right)=\left(V_{l}-\{0\}\right) / \boldsymbol{C}^{\wedge}$ and $\left[\eta_{l}(x)\right]=\eta_{l}(x) \bmod \boldsymbol{C}^{\times}$.

Lemma 3.5. For a graph $G$, there exists an isomorphism:

$$
\overline{L_{G}{ }^{\omega}} \cong \overline{\mathcal{L}_{G}{ }^{\omega}}
$$

where $\mathcal{L}_{G}{ }^{\omega}=\left\{\left[\eta_{l}(x)\right]_{l \in I_{0}} \in \prod_{l \in I_{0}} S\left(V_{l}\right) \mid x \in U^{\boldsymbol{R}}, \quad \eta_{l}(x)^{2}>0, \omega_{l}\left(\eta_{l}(x)\right)_{0}>0\right\}$. $S\left(V_{l}\right)$ is defind to be $\left(V_{l}^{\boldsymbol{R}}-\{0\}\right) / \boldsymbol{R}_{+}$.

Proof. As a representative of an element of $S\left(V_{l}\right)$ we take $k_{l}$ such that $k_{l}{ }^{2}=m_{l}{ }^{2}$. Then the mapping

$$
\begin{array}{rll}
\prod_{l \in I_{0}} S\left(V_{l}\right) & \rightarrow & \boldsymbol{R}^{n \nu} \\
U & & \Psi \\
{\left[k_{l}\right]_{l \in I_{0}}} & \mapsto & \left(\sum_{l \in I_{0}}[l: j] k_{l}\right)_{j=1, \ldots, n}
\end{array}
$$

is well-defined and maps $\mathcal{L}_{G}{ }^{\omega}$ into $L_{G}{ }^{\omega}$, hence $\overline{\mathcal{L}_{G}{ }^{\omega}}$ into $\overline{L_{G}{ }^{\omega}}$. The inverse mapping is given by Proposition 3.1.

In the above terminologies we have

$$
\operatorname{dim} \mathcal{L}_{G}{ }^{\boldsymbol{C}}=\rho(G)-\operatorname{dim}_{G} I_{0} .
$$

The key to calculate the mobility is the following theorem.

Theorem 3.6. Let $V$ be a finite dimensional vector space over a field $K$, and $V_{1}, \cdots, V_{N}$ its subspaces. We have

$$
\max _{\substack{x_{1} \in V_{1}, \cdots, x_{N} \in V_{N} \\ x_{1}+\cdots+x_{N}=0}} \operatorname{dim}\left(K x_{1}+\cdots+K x_{N}\right)
$$




$$
=\min _{I_{0}=I_{1} \cup \ldots \cup I_{k}}\left(N-k+\operatorname{dim} V_{I_{0}-I_{1}} \cap \cdots \cap V_{I_{0}-I_{k}}\right)
$$

where $V_{I}$ denotes the sum $\sum_{l \in I} V_{l}$.

Taking $V_{N+1}=V$ we have the

\section{Corollary 3.7 .}

$$
\max _{x_{1} \in V_{1}, \cdots x_{N} \in V_{N}}\left(K x_{1}+\cdots+K x_{N}\right)=\min _{I \subset I_{0}}\left(N-\# I+\operatorname{dim} V_{I}\right) .
$$

A proof of Theorem 3.6 is given in Sato, Miwa and Jimbo [12] and we omit it.

Theorem 3.8. (Dimension formula for a pseudo graph)

$$
\operatorname{dim} \mathcal{L}_{G}{ }^{\boldsymbol{c}}=\min _{\left.I_{0}=I_{1}\right\lrcorner \ldots \sqcup I_{k}} \sum_{k=1}^{k}\left(-1+\rho\left(G_{I_{k}}\right)\right)
$$

Proof. For a generic $x_{0}$ we have $\operatorname{dim}_{G, x_{0}} I_{0}=N-\operatorname{dim}\left(K D \eta_{1}\left(x_{0}\right)+\right.$ $\left.\cdots+K D \eta_{N}\left(x_{0}\right)\right)$, hence

$$
\operatorname{dim} I_{G} I_{0}=N-\max _{\substack{y_{1} \in W_{1}, \cdots, y_{N} \in W_{N} \\ y_{1}+\cdots, y_{N}=0}} \operatorname{dim}\left(K y_{1}+\cdots+K y_{N}\right)
$$

where $W_{l}=D V_{l}$. Applying Theorem 3.6 and (3.2), we have

$$
\operatorname{dim} \mathcal{L}_{G}{ }^{\boldsymbol{C}}=\rho(G)+\min _{I_{0}=I_{1} \sqcup \ldots \sqcup I_{I_{k}}}\left(-k+\operatorname{dim} W_{I_{0}-I_{1}} \cap \cdots \cap W_{I_{0}-I_{k}}\right) .
$$

Since we have $\operatorname{dim} W_{I_{0}-I_{1}} \cap \cdots \cap W_{I_{0}-I_{k}} \geqq-\sum_{k=1}^{k} \operatorname{dim} W_{I_{k}}+\operatorname{dim} W$,

$$
\begin{aligned}
\operatorname{dim} \mathcal{L}_{G} \boldsymbol{C} & \geqq \rho(G)+b_{1}(G)+\min \left(-\sum_{\kappa=1}^{k}\left(1+\operatorname{dim} W_{I_{k}}\right)\right) \\
& =\operatorname{dim} V+\min \left(\sum_{\kappa=1}^{k}\left(-1+\rho\left(G_{I_{k}}\right)-\operatorname{dim} V_{I_{\kappa}}\right)\right) \\
& =\min \sum_{\kappa=1}^{k}\left(-1+\rho\left(G_{I_{\kappa}}\right)\right) .
\end{aligned}
$$

Since there exists an injective mapping $\mathcal{L}_{G}^{\boldsymbol{C}} \rightarrow \prod_{\kappa=1}^{k} \mathcal{L}_{G_{I_{k}}}^{\boldsymbol{C}}$ and $\operatorname{dim} \mathcal{L}_{G_{I_{k}}}^{\boldsymbol{C}} \leqq$ $\rho\left(G_{I_{k}}\right)-1$ holds, the converse inequality is easy to see.

Note that the above theorem with Lemma 3.5 implies Theorem 2.3. 
Proposition 3.9. Let $G\left(U^{\eta_{l}} \rightarrow V_{l} \mid l \in I_{0}\right)$ and $\bar{G}\left(\bar{U}^{\bar{\eta}_{l}} \rightarrow V_{l} \mid l \in I_{0}\right)$ be two pseudo graphs, and assume that there exists a commutative diagram:

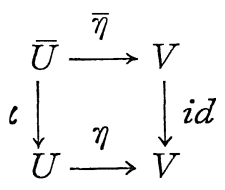

We assume that the induced mapping $\mathcal{L}_{\bar{G}}{ }^{\mathrm{C}} \rightarrow \mathcal{L}_{G}{ }^{\mathrm{c}}$ is generically surjective. Then for any generic element $x_{0} \in U$ and for any element $x \in U$ there exist $t \in K^{I_{0}}$ and $y \in \bar{U}$ such that

$$
\eta_{l}(x-\iota(y))=t_{l} \eta_{l}\left(x_{0}\right) \quad\left(l \in I_{0}\right) .
$$

Proof. From the assumption for any generic $x_{0} \in U$ we can take $t_{l} \neq 0 \quad\left(l \in I_{0}\right)$ such that $\sum_{l \in I_{0}} t_{l} \eta_{l}\left(x_{0}\right) \in \bar{\eta}(\bar{U})$.

In other words for any $l_{0} \in I_{0}$

$$
\begin{aligned}
\max _{x \in U} \operatorname{dim} & \left(\sum_{i \in I_{0}} K \eta_{l}(x)+\bar{\eta}(\bar{U})\right) / \bar{\eta}(\bar{U}) \\
= & \max _{x \in U} \operatorname{dim}\left(\sum_{\substack{l \in I_{0} \\
l \neq l_{0}}} K \eta_{l}(x)+\bar{\eta}(\bar{U})\right) / \bar{\eta}(\bar{U}) .
\end{aligned}
$$

On the other hand the conclusion is that for any $x \in U$ there exist $t_{l}$ 's such that $\eta(x)=\sum_{l \in I_{0}} t_{l} \eta_{l}\left(x_{0}\right) \bmod \bar{\eta}(\bar{U})$. In other words

$$
\begin{aligned}
\max _{x, x_{0} \in U} \operatorname{dim}\left(K \eta(x)+\sum_{l \in I_{0}} K \eta_{l}\left(x_{0}\right)+\bar{\eta}(\bar{U})\right) / \bar{\eta}(\bar{U}) \\
=\max _{x_{0} \in U} \operatorname{dim}\left(\sum_{l \in I_{0}} K \eta_{l}\left(x_{0}\right)+\bar{\eta}(\bar{U})\right) / \bar{\eta}(\bar{U}) .
\end{aligned}
$$

If we put $W_{0}=\eta(U) / \bar{\eta}(\bar{U})$ and $W_{l}=\left(V_{l}+\bar{\eta}(\bar{U})\right) / \bar{\eta}(\bar{U})$ and define

$$
\rho\left(W_{l_{1}}, \cdots, W_{l_{k}}\right)=\max _{\substack{w_{l_{1}} \in W_{l_{1}}, \cdots, w_{l_{k}} \in W_{l_{k}} \\ w_{l_{1}}+\cdots+w_{l_{k}}=0}} \operatorname{dim}\left(K w_{l_{1}}+\cdots+K w_{l_{k}}\right)
$$

we have

$$
\begin{aligned}
& \rho\left(W_{0}, W_{1}, \cdots, W_{N}\right)=\max _{x \in U} \operatorname{dim}\left(\sum_{i \in I_{0}} K \eta_{l}(x)+\bar{\eta}(\bar{U})\right) / \bar{\eta}(\bar{U}), \\
& \rho\left(W_{0}+W_{l_{0}}, W_{1}, \cdots, W_{l_{0}-1}, W_{l_{0}+1}, \cdots, W_{l_{k}}\right) \\
& =\max _{x \in U, \bar{x} \in \bar{U}} \operatorname{dim}\left(\sum_{\substack{l \in I_{0} \\
l \neq l_{0}}} K \eta_{l}(x)+\bar{\eta}(\bar{U})\right) / \bar{\eta}(\bar{U}), \\
& \rho\left(W_{0}, W_{0}, W_{1}, \cdots, W_{N}\right)
\end{aligned}
$$




$$
=\max _{x, x_{0} \in U} \operatorname{dim}\left(K \eta(x)+\sum_{l \in I_{0}} K \eta_{l}\left(x_{0}\right)+\bar{\eta}(\bar{U})\right) / \bar{\eta}(\bar{U}) .
$$

Then Proposition 3.9 is reduced to the following

Lemma 3.10. If $\rho\left(W_{0}, W_{1}, \cdots, W_{N}\right)=\rho\left(W_{0}+W_{l_{0}}, W_{1}, \cdots, W_{l_{0}-1}\right.$, $\left.W_{l_{0}+1}, \cdots, W_{N}\right)$ for any $l_{0} \in I_{0}$, we have $\rho\left(W_{0}, W_{0}, W_{1}, \cdots, W_{N}\right)=\rho\left(W_{0}, W_{1}\right.$, $\left.\cdots, W_{N}\right)$.

Proof. Taking $\tilde{I}_{0}=\{0,1, \cdots, N\}$ from Theorem 3.6 we have

$$
\begin{aligned}
\rho\left(W_{0}\right. & \left.+W_{l_{0}}, W_{1}, \cdots, W_{l_{0}-1}, W_{l_{0}+1}, \cdots, W_{N}\right) \\
& =\min _{\substack{\tilde{I}_{0}=I_{1} \sqcup \ldots \sqcup I_{1} \\
I_{1} \ni 0, l_{0}}}\left(N-k+\operatorname{dim} W_{\tilde{I}_{0}-I_{1}} \cap \cdots \cap W_{\tilde{I}_{0}-I_{k}}\right) .
\end{aligned}
$$

Theorem 3.6 implies also that there exists a partition $\widetilde{I}_{0}=\widetilde{I}_{1} \sqcup \cdots \sqcup \widetilde{I}_{k_{0}}$ $\left(\tilde{I}_{1} \ni 0\right)$ such that

$$
\rho\left(W_{0}, W_{1}, \cdots, W_{N}\right)=N+1-k_{0}+\operatorname{dim} W_{\tilde{I}_{0}-\tilde{I}_{1}} \cap \cdots \cap W_{\tilde{I}_{0}-\tilde{I}_{k_{0}}} .
$$

From the assumption these two coincide, hence we have $\widetilde{I}_{1}=\{0\}$. If we put $W_{N+1}=W_{0}$ and $I_{0}^{\prime}=\{0,1, \cdots, N+1\}$ and again make use of Theorem 3.6 we have

$$
\begin{aligned}
\rho\left(W_{0}, W_{1}, \cdots, W_{N}\right) & =N+2-\left(k_{0}+1\right) \\
& +\operatorname{dim} W_{I_{0^{\prime}}-\{0\}} \cap W_{I_{0^{\prime}}-\{N+1\}} \cap W_{I_{0^{\prime}}-\tilde{I}_{2}} \cap \cdots \cap W_{I_{0^{\prime}-\tilde{I}_{k}}} \\
& \geqq \rho\left(W_{0}, W_{1}, \cdots, W_{N+1}\right) .
\end{aligned}
$$

Since $\rho\left(W_{0}, W_{1}, \cdots, W_{N}\right) \leqq \rho\left(W_{0}, W_{0}, W_{1}, \cdots, W_{N}\right)$ is trivial, we have completed the proof.

Making use of Proposition 3.9, we investigate the properties of maximal primary subgraphs.

Definition 3.11. If $I \subset I_{0}$ is a maximal subset in those which have the same $\operatorname{dim}_{G, x_{0}} I$, then we say that $I$ is $\left(G, x_{0}\right)$-closed. If $I \subset I_{0}$ is $\left(G, x_{0}\right)$-closed for a generic element $x_{0} \in U$, we say that $I$ is $G$-closed or simply closed.

Proposition 3.12. I is closed if and only if the natural inclusion 


$$
\mathcal{L}_{G / I}^{\boldsymbol{C}} \hookrightarrow \mathcal{L}_{G_{I_{0}-I}}^{\boldsymbol{C}}
$$

is an isomorphism.

Proof. Easy.

Proposition 3.13. For $\left.I^{\prime} \subset I \subset I_{0}, \mathrm{i}\right)$ is equivalent to ii) plus iii).

i) I is G-closed

ii) $I^{\prime}$ is $G_{I_{0}-\left(I-I^{\prime}\right)}$-closed

iii) $I-I^{\prime}$ is $G / I^{\prime}$-closed

Proof. Straightforward from Proposition 3.12.

Proposition 3.14. Let $I$ be closed and $x_{1}$ be any element in $U$. For a generic element $x$ in $U_{x_{1}}\left(G_{I}\right)$, we have

$$
\operatorname{dim}_{G, x} I_{0}=\operatorname{dim}_{G_{I}, x} I+\operatorname{dim}_{G / I}\left(I_{0}-I\right) .
$$

Proof. Let $\bar{x}$ be a generic point of $U_{x}\left(G_{I_{0}-I}\right) \cap U_{G / I}$, then we have

$$
\operatorname{dim}_{G, x} I_{0}=\operatorname{dim}_{G, x} I+\operatorname{dim}_{G / I, \bar{x}}\left(I_{0}-I\right) .
$$

Since $I$ is closed, $\mathcal{L}_{G / I}^{\boldsymbol{C}} \rightarrow \mathcal{L}_{G_{I_{0}-I}}^{\boldsymbol{C}}$ is an isomorphism and we can apply Proposition 3.9. Thus for a generic $x_{0} \in U$, there exist $y \in U_{G / I}$ and $t_{l} \neq 0$ such that

$$
\eta_{l}\left(x_{1}-y\right)=t_{l} \eta_{l}\left(x_{0}\right) \quad\left(l \in I_{0}-I\right) .
$$

This implies that $\left[\eta_{l}(x)\right]_{l \in I_{0}-I}$ have not been restricted by the condition $x \in U_{x_{1}}\left(G_{I}\right)$. Therefore $\eta_{l}(\bar{x})\left(l \in I_{0}-I\right)$ have not been restricted by the condition $\bar{x} \in U_{x}\left(G_{I_{0}-I}\right)$. Thus we have

$$
\operatorname{dim}_{G / I, \bar{x}}\left(I_{0}-I\right)=\operatorname{dim}_{G / I}\left(I_{0}-I\right) .
$$

Again using Proposition 3.9, we can show that for any $x^{\prime} \in U_{x}\left(G_{I}\right)$ there exist $y^{\prime} \in U_{G / I}$ and $t_{l}{ }^{\prime} \neq 0\left(l \in I_{0}-I\right)$ such that

$$
\eta_{l}\left(x^{\prime}-y^{\prime}\right)=t_{l}{ }^{\prime} \eta_{l}\left(x_{0}\right) \quad\left(l \in I_{0}-I\right) \text {. }
$$

Since $\left[\eta_{l}(x)\right]_{l \in I_{0}-I}$ have not been restricted, this implies that we can choose $y^{\prime} \in U_{G / I}$ such that $x^{\prime}-y^{\prime} \in U_{x}(G)$ and hence

$$
\operatorname{dim}_{G_{I}, x} I=\operatorname{dim}_{G, x} I .
$$


$(3 \cdot 4),(3 \cdot 5)$ and $(3 \cdot 6)$ imply $(3 \cdot 3)$.

\section{Corollary 3.15.}

i) If $I$ is closed, $\operatorname{dim}_{G} I=\operatorname{dim}_{G_{I}} I$.

ii) I is closed if and only if

$$
\operatorname{dim}_{G} I_{0}=\operatorname{dim}_{G_{I} I} I+\operatorname{dim}_{G / I}\left(I_{0}-I\right) .
$$

iii) If $I$ is closed and $I \supset I^{\prime}$, then

$$
\operatorname{dim}_{G} I_{0}-\operatorname{dim}_{G / I^{\prime}}\left(I_{0}-I^{\prime}\right)=\operatorname{dim}_{G_{I}} I-\operatorname{dim}_{G_{I^{\prime} / I^{\prime}}}\left(I-I^{\prime}\right) .
$$

Proof. Taking $x_{1}$ to be generic in $(3 \cdot 3)$, we have

$$
\operatorname{dim}_{G} I_{0}=\operatorname{dim}_{G_{I}} I+\operatorname{dim}_{G / I}\left(I_{0}-I\right) .
$$

In general the following holds

$$
\operatorname{dim}_{G} I_{0} \leqq \operatorname{dim}_{G} I+\operatorname{dim}_{G / I}\left(I_{0}-I\right) \leqq \operatorname{dim}_{G_{I}} I+\operatorname{dim}_{G / I}\left(I_{0}-I\right) .
$$

Hence i) and the necessity of $(3 \cdot 7)$ are proved. To prove the sufficiency of $(3 \cdot 7)$, take a closed subset $\bar{I}$ such that

$$
\operatorname{dim}_{G} \bar{I}=\operatorname{dim}_{G} I \text { and } \bar{I} \supset I .
$$

Then we have

$$
\operatorname{dim}_{G} I_{0}=\operatorname{dim}_{G} \bar{I}+\operatorname{dim}_{G / I}\left(I_{0}-\bar{I}\right),
$$

hence

$$
\operatorname{dim}_{G / I}\left(I_{0}-I\right)=\operatorname{dim}_{G / I}\left(I_{0}-\bar{I}\right) .
$$

By Proposition $3.13 \bar{I}-I$ is closed in $G / I$, hence

$$
\operatorname{dim}_{G / I}\left(I_{0}-I\right)=\operatorname{dim}_{G / I}(\bar{I}-I)+\operatorname{dim}_{G / I}\left(I_{0}-\bar{I}\right) .
$$

From $(3 \cdot 8)$ and $(3 \cdot 9)$ we have $\operatorname{dim}_{G / I}(\bar{I}-I)=0$. This implies that $I=\bar{I}$.

It is easy to see that Proposition (3.13) and (3.7) imply iii).

Definition 3.16 $I$ is called maximal r-ary if the following two conditions are satisfied:

i) $\operatorname{dim}_{G_{I}} I \leqq r$,

ii) If there exist $I^{\prime}$ such that $I \subset I^{\prime}$ and $\operatorname{dim}_{G_{I}} I^{\prime} \leqq \operatorname{dim}_{G_{I}} I^{\prime}$, then $I=I^{\prime}$.

Note that when $G$ is a graph and $r=1$, the above definition coincides with the definition of a maximal primary subgraph.

Proposition 3.17. I is closed if and only if $I$ is maximal r-ary 
for some $r$.

Proof. Easy.

Especially a maximal primary subgraph is closed. Then it is easy to see that iii) of Corollary 3.15 implies Theorem 2.9.

Theorem 3.18. Let $G$ be a graph and I be a closed subset. Take an orientation $\omega$ of $G$ which is compatible with a contraction of $I$. Then we have an isomorphism

$$
\overline{L_{G}{ }^{\omega}} \cong \overline{L_{G I}^{\omega}} \times \overline{L_{G / I}^{\omega}}
$$

Proof. Let $p$ be a generic element of $L_{G}{ }^{\omega}$. We take a generic configuration $x$ of $p$. Since $I$ is closed, we can take $\bar{x}$ and $t_{l} \neq 0$ ( $l$ $\left.\in I_{0}-I\right)$ such that $\eta_{l}(\bar{x})=t_{l} \eta_{l}(x)\left(l \in I_{0}-I\right)$ and $\eta_{l}(\bar{x})=0(l \in I)$. This implies $\bar{p}$ defined by

$$
\bar{p}_{j}=\sum_{l \in \tau_{T^{-1}}(j)} p_{i}
$$

is in $L_{G / I}^{\omega}$. It is obvious that $p_{I}$ defined by

$$
p_{I j}=p_{j}-\sum_{l \in I_{0}-I}[l: j] k_{l}^{G}(p)
$$

is in $L_{G_{I}}^{\omega}$. Thus $(3 \cdot 10)$ and $(3 \cdot 11)$ give a mapping

$$
\overline{L_{G}{ }^{\omega}} \longrightarrow \overline{L_{G_{I}}^{\omega}} \times \overline{L_{G / I}^{\omega}}
$$

Now take generic elements $\left(p_{I}, x_{I}\right) \in \Lambda_{G_{I}}^{\omega}$ and $(\bar{p}, \bar{x}) \in \Lambda_{G / I}^{\omega}$. From Proposition 3.12, there is a generic configuration $x_{I_{0}-I}$ of $G_{I_{0}-I}$ such that $\eta_{l}\left(x_{I_{0}-I}\right)$ $\| \eta_{l}(\bar{x})\left(l \in I_{0}-I\right)$. Applying Proposition 3.9 to the case where $\bar{G}=G / I$, $G=G_{I_{0}-I}, x_{0}=x_{I_{0}-I}$ and $x=x_{I}$, we can easily see that there exist a configuration $x$ of $G$ such that $\eta_{l}(x)=t_{l} \eta_{l}\left(x_{I}\right)(l \in I)$ and $\eta_{l}(x)=t_{l} \eta_{l}(\bar{x})$ $\left(l \in I_{0}-I\right)$ for some $t_{l} \neq 0$. Thus if we define $p$ by

$$
p_{j}=p_{I j}+\sum_{l \in I_{0}-I}[l: j] k_{l}^{G / I}(\bar{p}),
$$

$p$ is in $L_{G}{ }^{\omega}$ and $(3 \cdot 12)$ gives an inverse of $(3 \cdot 10)$ and $(3 \cdot 11)^{\circ}$

By the same argument we can prove the following 
Theorem 3.19. For a closed subset I there exists an isomorphism

$$
\overline{L_{G}{ }^{\omega}} \cong \overline{L_{G_{I}}^{\omega}} \times \overline{L_{G_{I 0-I}}^{\omega}}
$$

For a maximal primary partion $I_{0}=I_{1} \sqcup \cdots \sqcup I_{k}$, teere exists an isomorphism

$$
\overline{L_{G}{ }^{\omega}} \stackrel{\cong}{\Longrightarrow} \overline{L_{G_{I_{1}}}^{\omega}} \times \cdots \times \overline{L_{G_{I_{k}}}^{\omega}}
$$

\section{§ 4. Oridinary Components of Non External Graphs}

In this section we consider non external graphs and obtain several topological formulas for an ordinary component of $\Lambda_{G, \sigma}^{\boldsymbol{C}}$.

If we consider general Feynman graphs with internal vertices, the Landau variety $\Lambda_{G, \sigma}^{\mathcal{G}}$ is no longer irreducible, nor is it manifestly holonomic as in the external case. Henceforth we regard the masses of internal lines as variables and introduce $\mu_{l}=-\frac{1}{2} m_{l}{ }^{2}$, and its dual variable $\alpha_{l}$. Thus a Landau holonomic variety $\Lambda_{G, \sigma}^{\boldsymbol{C}}$ is a subvariety of $(p, \mu ; x, \alpha)$-space $T^{*} C^{\nu n+N}$ and similarly for $\Lambda_{G, \sigma}^{+}, \Lambda_{G, \sigma}^{\omega}$ etc.

In what follows as for notations of graph theory we follow Nakanishi [8]. Especially function $U_{G}$ of Nakanishi play an important role.

Let $G$ be a connected external graph, and $J$ be a non empty subset of its vertices $J_{0}$. We use the following notations.

$$
\begin{aligned}
& J^{*}=J_{0}-J, \\
& x_{J}=\left(x_{j}\right)_{j \in J}, \quad p_{J}=\left(p_{j}\right)_{j \in J}, \quad x_{J^{*}}=\left(x_{j^{*}}\right)_{j^{*} \in J^{*}}, \\
& p_{J^{*}}=\left(p_{j^{*}}\right)_{j^{*} \in J^{*}}, \quad \alpha=\left(\alpha_{l}\right)_{l \in I_{0}}, \quad \mu=\left(\mu_{l}\right)_{l \in I_{0}} .
\end{aligned}
$$

We also denote by $G[J$ the graph obtained by identifying all the vertices in $J$. In $G\left[J\right.$ the vertex thus obtained is denoted by $j_{0}$. We fix a reference tree $T$ in $G[J]$. Then $j^{*} \in J^{*}$ determines a path on $T$ from $j_{0}$ to $j^{*}$. We denote this path by $P_{j^{*}}$ and the incidence number of $P_{j^{*}}$ by $\left[P_{j^{*}}: l\right]$. Let $T^{*}$ be the cotree of $T$, that is, $T^{*}=I_{0}-T$. Then $l^{*} \in T^{*}$ determines a circuit $C_{l^{*}}$ in $G[J]$ which is characterized by $C_{l^{*}} \cap T^{*}=\left\{l^{*}\right\}$.

We define $z_{C_{l^{*}}}\left(x_{J}\right)=\sum_{l \in I_{0}, j \in I_{0}}\left[C_{l^{*}}: l\right][l: j] x_{j}$, which is zero if $C_{l^{*}}$ is also a circuit in $G$ and $x_{j_{1}}-x_{j_{2}}$ if $C_{l^{*}}$ is a path from $j_{2}$ to $j_{1}$ in $G$.

Now consider the Landau equations for $G$. The energy-momentum 
conservation gives for $l \in T$

$$
k_{l}=\sum_{l^{*} \in I^{*}}\left[C_{l^{*}}: l\right] k_{l^{*}}+\sum_{j^{*} \in J^{*}}\left[P_{j^{*}}: l\right] p_{j^{*}} .
$$

The circuit condition gives for $l^{*} \in T^{*}$

$$
\sum_{l \in I_{0}}\left[C_{l^{*}}: l\right] \alpha_{l} k_{l}=z_{c^{*}}\left(x_{J}\right) .
$$

Following Nakanishi we define

$$
X_{l l^{\prime}}(\alpha)=\sum_{C} \frac{U_{G[J] / C}(\alpha)}{U_{G[J]}(\alpha)}[C: l]\left[C: l^{\prime}\right]
$$

where the sum is over all the circuits in $G[J]$, and

$$
W_{l}^{\left(j^{*} \mid j_{0}\right)}(\alpha)=\sum_{P: j_{0} \rightarrow j^{*}}[P: l] U_{G[J] / P}(\alpha)
$$

where the sum is over all the paths from $j_{0}$ to $j^{*}$ in $G[J]$.

$(4 \cdot 1)$ and $(4 \cdot 2)$ gives

$$
\begin{aligned}
& \sum_{l k^{\prime} \in T^{*}}(\left.\sum_{l \in I_{0}}\left[C_{l^{*}}: l\right]\left[C_{l^{*}}: l\right] \alpha_{l}\right) k_{l^{* \prime}} \\
& \quad=z_{C_{l^{*}}}\left(x_{J}\right)-\sum_{j^{*} \in J^{*}}\left(\sum_{l \in I_{0}}\left[C_{l^{*}}: l\right]\left[P_{j^{*}}: l\right] \alpha_{l}\right) p_{j^{*}}
\end{aligned}
$$

From $(4 \cdot 1)$ and $(4 \cdot 3)$ we can express $k_{l}$ 's $\left(l \in I_{0}\right)$ as rational functions of $p_{J^{*}}, x_{J}$ and $\alpha$. Then we have the

Proposition 4.1. For $l \in T \cup T^{*}$ we have

$$
\begin{aligned}
& k_{l}\left(p_{J^{*}}, x_{J}, \alpha\right)=\sum_{j^{*} \in J^{*}} \frac{W_{l}^{\left(j^{*} \mid j_{0}\right)}(\alpha)}{U_{G[J]}(\alpha)} p_{j^{*}}+\sum_{j \in J} \sum_{l^{\prime} \in I_{0}} X_{l l^{\prime}}(\alpha)\left[l^{\prime}: j\right] x_{j}, \\
& \frac{\partial k_{l}}{\partial \alpha_{l^{\prime}}}\left(p_{J^{*}}, x_{J}, \alpha\right)=-X_{l l^{\prime}}(\alpha) k_{l^{\prime}}\left(p_{J^{*}}, x_{J}, \alpha\right) .
\end{aligned}
$$

Proof. First suppose that $l$ is not a cut line. Then we can choose $T$ so that $l \in T^{*}$ and differentiating $(4 \cdot 3)$ by $p_{j^{*}}$ we have

$$
\frac{\partial k_{l^{*}}}{\partial p_{j^{*}}}=-\sum_{l^{*} \in T^{*}}\left(U(\alpha)^{-1}\right)_{l^{*} l^{*}} \sum\left[C_{l^{*}}: l\right]\left[P_{j^{*}}: l\right] \alpha_{l} I_{\nu}
$$

We have used $l^{*}$ instead of $l$, and $U(\alpha)=\left(\sum\left[C_{l^{*}}: l\right]\left[C_{l^{*}}: l\right] \alpha_{l}\right)_{l^{*}, l^{*} \in T^{*}}$. Note that $\frac{\partial k_{l^{*}}}{\partial k_{j^{*}}}$ is a $\nu \times \nu$ matrix and $I_{\nu}$ is the unit matrix. Then from 
p. 78 of Nakanishi [8] we have

$$
\frac{\partial k_{l^{*}}}{\partial p_{j^{*}}}=\frac{W_{l^{*}}^{\left(j^{*} \mid j_{0}\right)}(\alpha)}{U_{G[J]}(\alpha)} I_{\nu}
$$

Differentiating $(4 \cdot 3)$ by $x_{j}$ and using (7-29) of Nakanishi [8] we have

$$
\frac{\partial k_{l^{*}}}{\partial x_{j}}=\sum_{l^{\prime}}\left[l^{\prime}: j\right] X_{l^{*} l^{\prime}}(\alpha) .
$$

Thus when $l$ is not a cut line, we have proved (4.4).

To prove $(4 \cdot 5)$ first assume that we can take $T$ so that $l=l^{*}$, $l^{\prime}=l^{* \prime} \in T^{*}$. Differentiating $(4 \cdot 3)$ by $\alpha_{l^{*}}$ we have

$$
\frac{\partial k_{l^{*}}}{\partial \alpha_{l^{* \prime}}}=-\left(U(\alpha)^{-1}\right)_{l^{*} l^{*}} k_{l^{* \prime}} .
$$

Again using (7-29) of Nakanishi [8] we have (4.5).

The case when $\left\{l, l^{\prime}\right\}$ is a cut set or $l$ is a cut line is easy.

Note that when $\#(J)=1,(4 \cdot 4)$ is nothing but Theorem $9-4$ of Nakanishi [8].

Theorem 4.2. $\Lambda_{G}^{c}$ is represented by a rational potential function

$$
\begin{aligned}
H\left(p_{J^{*}}, x_{J}, \alpha\right)= & \sum_{j \in J} x_{j} p_{j}\left(p_{J^{*}}, x_{J}, \alpha\right) \\
& +\sum_{l \in I_{0}} \alpha_{l} \mu_{l}\left(p_{J^{*}}, x_{J}, \alpha\right)
\end{aligned}
$$

as

$$
\begin{gathered}
\Lambda_{G}{ }^{c}=\text { closure of }\left\{(p, \mu ; x, \alpha) \mid x_{J^{*}}=-\frac{\partial H}{\partial p_{J^{*}}},\right. \\
\left.p_{J}=\frac{\partial H}{\partial x_{J}}, \mu=\frac{\partial H}{\partial \alpha}, \alpha_{l} \neq 0, U_{G[J]}(\alpha) \neq 0\right\} .
\end{gathered}
$$

Proof. $\Lambda_{G}^{C}$ is given by

$$
\begin{aligned}
& x_{j^{*}}\left(p_{J^{*}}, x_{J}, \alpha\right)=x_{j_{0}}+\sum_{l}\left[P_{j^{*}}: l\right] \alpha_{l} k_{l}\left(p_{J^{*}}, x_{J}, \alpha\right), \\
& p_{j}\left(p_{J^{*}}, x_{J}, \alpha\right)=\sum_{l}[l: j] k_{l}\left(p_{J^{*}}, x_{J}, \alpha\right), \\
& \mu_{l}\left(p_{J^{*}}, x_{J}, \alpha\right)=-\frac{1}{2} k_{l}\left(p_{J^{*}}, x_{J}, \alpha\right)^{2} .
\end{aligned}
$$


The above theorem follows from the following

Lemma 4.3. Let us devide coordinates in $C^{n}$ into $(x, y)$. Let $(\xi, \eta)$ be their dual variables. Assume that the image of a subvariety $\Lambda$ in $T^{*} C^{n}$ into $(x, \eta)$ coordinates is generically surjective. Then $\Lambda$ is holonomic if and only if it can be expressed as

$$
\Lambda=\left\{(x, y ; \xi, \eta) \mid y=\frac{\partial H}{\partial \eta}, \xi=-\frac{\partial H}{\partial x}\right\}
$$

by a suitable analytic function $H(x, \eta)$ which is homogeneous of degree 1 with respect to $\eta$. $H(x, \eta)$ is given by

$$
H(x, \eta)=\eta \cdot y(x, \eta) .
$$

Proof. Easy.

Let us consider a graph $\left(G, J_{0}, \sigma\right)$ whose set of internal (external) vertices is $J_{i n t}\left(J_{e x t}\right)$. Take a non empty subset $J \subset J_{\text {ext }}$, and consider $H\left(p_{J^{+}}, x_{J}, \alpha\right)$ with $G$ as an external graph. Then the holonomic variety given by a potential function $H\left(0, p_{J^{*}-J i n t}, x_{J}, \alpha\right)$ is an irreducible component of $\Lambda_{G, \sigma}^{\boldsymbol{C}}$. This component is a unique one whose projection to $\alpha$ space is generically surjective.

Definition 4.3. We call the above component an ordinary component and denote by $\Lambda_{G \text {,ord }}^{\boldsymbol{C}}$.

Proposition 4.4. All the irreducible components other than $\Lambda_{G \text {,ord }}^{\boldsymbol{C}}$ are contained in $\bigcap_{J}\left\{U_{G[J]}(\alpha)=0\right\}$ when projected into $\alpha$ space, where $J$ runs over nonempty subsets of external vertices of $(G, \sigma)$.

We remark that $\Lambda_{G, \sigma}^{+} \subset \Lambda_{G \text {,ord }}^{C}$ holds, because $U$-functions are strictly positive on the interior of $\Lambda_{G, \sigma}^{+}$. Another important property of $\Lambda_{G \text {, ord }}^{C}$ is that, on $\Lambda_{G \text {,ord }}^{\boldsymbol{C}}$ the $k_{l}$ 's are linearly dependent on the $p_{j}$ 's, and so are the $y_{i}$ 's (the position vectors of internal vertices) on the $x_{j}$ 's (external ones).

Example 4.5. (Fig. (3.1))

$\Lambda_{G}^{C}$ has 2 components (i) $\Lambda_{G \text {,ord, }}^{\boldsymbol{C}}$, where all the $k_{l}$ 's are parallel, and (ii) one that has a 2 -dimensional realization. 


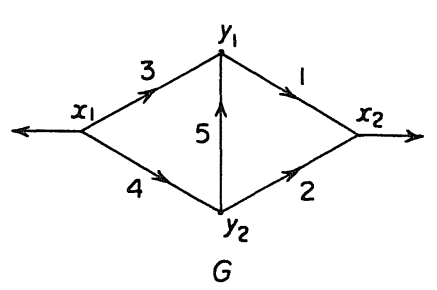

Fig. (3.1)

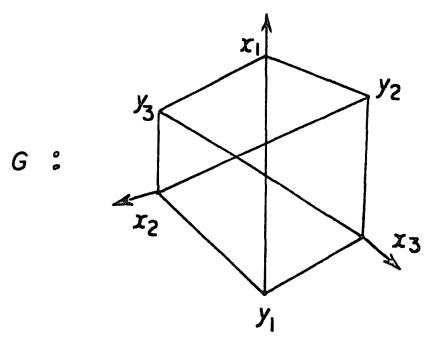

Fig. (3.2)

Example 4.6. (Fig. 3.2)

There are 6 components. $(\nu \geqq 4)$

(i) $\Lambda_{G \text {, ord }}^{\boldsymbol{C}} 2$-dimensional realization.

(ii) $\sim$ (iv)

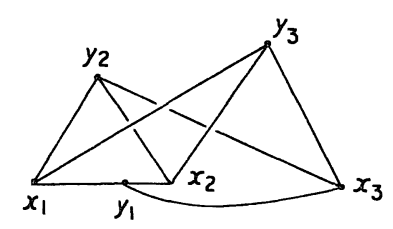

(v)

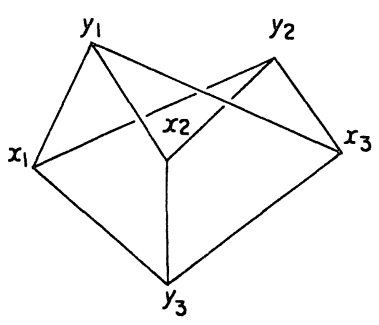

(vi)

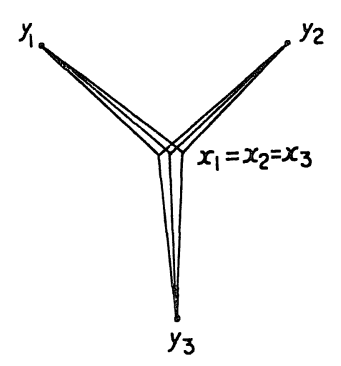

3-dimensional realization $x_{1}-y_{1}, x_{2}-y_{1}$ and $x_{3}-y_{1}$ are collinear.

4-dimensional realization. $x_{2}-x_{1}$ and $x_{3}-x_{1}$ are collinear.

3-dimensional realization.

All these components have rational potential functions with respect to suitably chosen subset of variables $(p, \mu ; x, \alpha)$. We conjecture that this is true for any graph $G$.




ses as fixed parameters, the leading Landau variety $\Lambda_{G \text {, ord }}^{C}$ is not necessarily present. For example, in the case of Fig. (3.3), $\Lambda_{G \text {, ord }}^{\boldsymbol{C}}$ is present if and only if $m_{1}+m_{2}=m_{3}+m_{4} \cdot{ }^{(\dagger)}$



Fig. (3.3)

Take a potential function $H(x, \alpha)$ where $x$ is the position vector of all the external vertices, and denote by $\pi_{\mu}$ the projection $T^{*} \mathbb{C}^{\nu n+N} \rightarrow \mathbb{C}^{N}$ to the $\mu$-space. Then

$$
\operatorname{codim} \pi_{\mu}\left(\Lambda_{G, \text { ord }}^{C}\right)=N-\operatorname{rank}\left(\frac{\partial^{2} H}{\partial \alpha \partial x}, \frac{\partial^{2} H}{\partial \alpha \partial \alpha}\right),
$$

because $\mu_{\iota}=\frac{\partial H}{\partial \alpha_{l}}$ holds .

\section{Theorem 4.7.}

$$
\begin{aligned}
N- & \operatorname{rank}\left(\frac{\partial^{2} H}{\partial \alpha \partial x}, \frac{\partial^{2} H}{\partial \alpha \partial \alpha}\right) \\
& =\operatorname{corank}\left(\frac{\partial^{2} H}{\partial \alpha \partial \alpha}\right) \\
& =\text { the freedom of } \alpha_{l}^{\prime} \text { 's with generically fixed } k_{l}^{\prime} \text { 's and } x_{j}^{\prime} \text { s. }
\end{aligned}
$$

This follows from the topological formula for Hessian of $H$ :

+ On the other hand, if we consider in the positive- $\alpha$ region, $\Lambda_{\theta}^{+} \sigma$ may be absent for special values of the masses as in the case of Fig. (3.4) with $m_{1}+m_{2}=m_{3}+m_{4}$ (communicated by Professor Kawai)

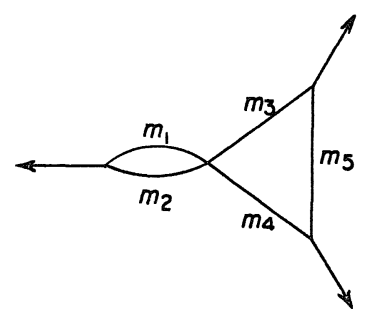

Fig. (3. 4) 
Theorem 4.8.

$$
\begin{aligned}
{ }^{t}\left(\widetilde{x}^{\prime}, \tilde{\alpha}^{\prime}\right)\left(\begin{array}{ll}
\frac{\partial^{2} H}{\partial x \partial x} & \frac{\partial^{2} H}{\partial x \partial \alpha} \\
\frac{\partial^{2} H}{\partial \alpha \partial x} & \frac{\partial^{2} H}{\partial \alpha \partial \alpha}
\end{array}\right)\left(\begin{array}{l}
\tilde{x} \\
\tilde{\alpha}
\end{array}\right) & =\sum_{C} \frac{U_{G\left[J_{\mathrm{ext}}\right] / C}}{U_{G\left[J_{\mathrm{ext}}\right]}} \times \\
& \times \eta_{C}(x, \alpha ; \tilde{x}, \tilde{\alpha}) \eta_{C}\left(x, \alpha ; \tilde{x}^{\prime}, \tilde{\alpha}^{\prime}\right)
\end{aligned}
$$

Here $\eta_{C}(x, \alpha ; \tilde{x}, \widetilde{\alpha})=\sum_{l}[C: l]\left(\widetilde{\alpha}_{l} k_{l}(x, \alpha)\right)-\sum_{j}[l: j] \widetilde{x}_{j}$, and $C$ runs over all the circuits in $G\left[J_{e x t}\right]$.

Proof of Theorem 4.7. Let us consider in the region where $\alpha_{l}>0$ and $k_{l} \in \boldsymbol{R} \times \sqrt{-1} \boldsymbol{R}^{\nu-1}$. From Theorem 4.8 we have

$$
\sum_{l, l^{\prime}} \frac{\partial^{2} H}{\partial \alpha_{l} \partial \alpha_{l^{\prime}}} \widetilde{\alpha}_{l} \widetilde{\alpha}_{l^{\prime}}=\sum_{C} \frac{U_{G\left[J_{\mathrm{ex}}\right] / C}}{U_{G\left[J_{\mathrm{ex} t}\right]}}\left(\sum_{l}[C: l] \widetilde{\alpha}_{l} k_{l}(x, \alpha)\right)^{2}
$$

which implies

$$
\begin{aligned}
\operatorname{Ker}\left(\frac{\partial^{2} H}{\partial \alpha \partial \alpha}(x, \alpha)\right)= & \left\{\widetilde{\alpha} \mid \sum_{l}[C: l] \widetilde{\alpha}_{l} k_{l}(x, \alpha)=0\right. \\
& \text { for any circuit in } \left.G\left[J_{\text {ext }}\right]\right\} .
\end{aligned}
$$

This is just the latter equality of Theorem 4.7.

The former equality is equivalent to

$$
\operatorname{rank}\left(\frac{\partial^{2} H}{\partial \alpha \partial x}, \frac{\partial^{2} H}{\partial \alpha \partial \alpha}\right)=\operatorname{rank}\left(\frac{\partial^{2} H}{\partial \alpha \partial \alpha}\right)
$$

From Theorem 4.8

$$
{ }^{t}(\widetilde{\alpha})\left(\frac{\partial^{2} H}{\partial \alpha \partial x}, \frac{\partial^{2} H}{\partial \alpha \partial \alpha}\right)\left(\begin{array}{c}
\widetilde{x}^{\prime} \\
\tilde{\alpha}^{\prime}
\end{array}\right)=\sum_{C} \frac{U_{G\left[J_{\mathrm{ext}}\right] / C}}{U_{G\left[J_{\mathrm{ext}}\right]}} \widetilde{z}_{C \widetilde{y} C^{\prime}}
$$

where

$$
\widetilde{z}_{C}=\sum_{l}[C: l] \widetilde{\alpha}_{l} k_{l}(x, \alpha)
$$

and

$$
\widetilde{y}^{\prime}=\sum_{l}[C: l]\left(\widetilde{\alpha}_{l}{ }^{\prime} k_{l}(x, \alpha)-\sum_{j}[l: j] x_{j}{ }^{\prime}\right) .
$$

If $\tilde{\alpha} \in \operatorname{Ker}\left(\frac{\partial^{2} H}{\partial \alpha \partial \alpha}\right)$ then $\widetilde{z}_{C}=0$ and $(4 \cdot 7)$ vanishes. This implies 


$$
{ }^{t}(\widetilde{\alpha})\left(\frac{\partial^{2} H}{\partial \alpha \partial x}, \frac{\partial^{2} H}{\partial \alpha \partial \alpha}\right)=0
$$

and $(4 \cdot 6)$ is proved.

Proof of Theorem 4.8. Straightforward calculation from Proposition 4.1.

We will call a graph $(G, \sigma)$ Lendauian if the $\alpha_{l}$ 's are uniquely

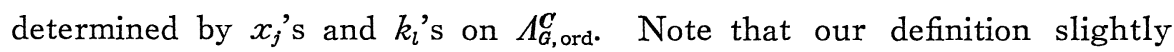
differs from that of Speer and Westwater [17].

If we regard the $m_{l}$ 's as fixed parameters, the Landau variety for the graph of Fig. (3.3) coincides with the one for the following Landauian graph if $m_{1}+m_{2}=m_{3}+m_{4}$.

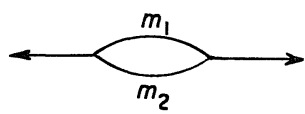

Fig. (3.5)

In general, for a non Landauian graph $(G, \sigma)$, we may always find a (not necessarily unique) Landauian graph $\left(G_{1}, \sigma_{1}\right)$ such that $\Lambda_{G, \sigma, \text { ord }}^{C}=\Lambda_{G_{1}, \sigma_{1}, \text { ord }}^{C}$ holds. Therefore it suffices to consider only Landauian graphs as far as the location of Landau singularity is concerned. Theorem 4.8 implies the

Proposition 4.9. The following conditions are equivalent:

(i) $(G, \sigma)$ is Landauian.

(ii) $\Lambda_{G \text {,ord }}^{\boldsymbol{C}}$ is present for generically fixed values of the masses.

(iii) $\operatorname{rank}\left(\frac{\partial^{2} H}{\partial \alpha \partial \alpha}\right)=N$.

In particular if codim $L_{G \text {, ord }}^{\boldsymbol{C}}$ in $\boldsymbol{C}^{\nu n+N}$ is equal to $\nu b_{0}(G)+1(G, \sigma)$ is necessarily Landauian, for otherwise at least one constraint among the masses is added. Finally Proposition 3.1 has an extention of the following form: 
Proposition 4.10. (cf. Speer-Westwater [15] Lemma 4.1.4.) The $k_{l}$ 's are rational functions on the irreducible algebric variety $L_{G \text {, ord. }}^{C}$.

Proof. We assume that $\operatorname{codim} L_{G \text {, ord }}^{\boldsymbol{C}}=\nu+s$. Fix an external vertex $j_{0}$. Then on $\Lambda_{G \text {,ord }}^{\boldsymbol{C}} k_{l}$ 's are rational functions of $\alpha_{l}$ 's and $p_{j}$ 's $\left(j \neq j_{0}\right)$. At a generic point on $\Lambda_{G}{ }^{c}$, corank $\partial\left(k_{1}{ }^{2}, \cdots, k_{N}{ }^{2}\right) / \partial\left(\alpha_{1}, \cdots, \alpha_{N}\right)=s$. From Proposition $4.1 \partial\left(k_{1}{ }^{2}, \cdots, k_{N}{ }^{2}\right) / \partial\left(\alpha_{1}, \cdots, \alpha_{N}\right)$ is symmetric, hence without loss of generality we may assume that

$$
\operatorname{det} \partial\left(k_{s+1}^{2}, \cdots, k_{N}^{2}\right) / \partial\left(\alpha_{s+1}, \cdots, \alpha_{N}\right) \neq 0 .
$$

Thus on $\Lambda_{G}{ }^{C} \alpha_{s+1}, \cdots, \alpha_{N}$ can be solved with respect to $\alpha_{1}, \cdots, \alpha_{s}, \mu_{s+1}$, $\cdots, \mu_{N}, p_{j}\left(j \neq j_{0}\right)$. Hence $\mu_{1}, \cdots, \mu_{s}$ also depend upon these variables. If we fix $\mu$ and $p$, we can vary $\alpha_{1}, \cdots, \alpha_{s}$ independently. This means that $\mu_{1}, \cdots, \mu_{s}$ depend only upon $\mu_{s+1}, \cdots, \mu_{N}, p_{j}\left(j \neq j_{0}\right)$ on $L_{G \text {,ord. }}^{\boldsymbol{C}}$ Thus $\mu_{s+1}, \cdots$, $\mu_{N}, p_{j}\left(j \neq j_{0}\right)$ form a local coordinate system on $L_{G \text {, ord. }}^{\boldsymbol{C}}$.

Following Speer-Westwater define

$$
\begin{gathered}
U=\text { closure of }\left\{(p, \mu, \alpha) \in \mathbb{C}^{\nu n+2 N} \mid\right. \\
\left.\frac{\partial V}{\partial \alpha_{l}}=0, \alpha_{l} \neq 0, U_{G}(\alpha) \neq 0\right\}
\end{gathered}
$$

where

$$
V(p, \mu, \alpha)=\sum_{j, j^{\prime} \neq j_{0}} \frac{U_{G\left[\left\{j, j^{\prime}\right\}\right]}}{U_{G}} p_{j} \cdot p_{j^{\prime}}+2 \sum_{l} \alpha_{l} \mu_{l}
$$

It is well-known that the projection to $(p, \mu)$-space of $U$ is $L_{G \text {,ord. }}^{C}$. From the homogeneity $\left.V\right|_{U}=0$, hence

$$
d_{p, \mu} V=d V-\sum_{i} \frac{\partial V}{\partial \alpha_{l}} d \alpha_{l}
$$

vanishes on $U$.

At a generic point $L_{G \text {,ord }}^{\boldsymbol{C}}$ can be expressed as

$$
L_{G, \text { ord }}^{\boldsymbol{C}}=\left\{(p, \mu) \in \mathbb{C}^{\nu n+N}: \sum_{j=1}^{N} p_{j}=0, f_{1}=\cdots=f_{s}=0\right\}
$$

where $f_{i}(p, \mu)$ 's are polynomials of $p$ and $\mu$ such that $\operatorname{det} \partial\left(f_{1}, \cdots, f_{s}\right) /$ $\partial\left(\mu_{1}, \cdots, \mu_{s}\right) \neq 0$. Then

$$
\Omega=\left(\alpha_{1}, \cdots, \alpha_{s}\right)\left(\frac{\partial\left(f_{1} \cdots f_{s}\right)}{\partial\left(\mu_{1} \cdots \mu_{s}\right)}\right)^{-1}\left(\begin{array}{c}
d f_{1} \\
\vdots \\
d f_{s}
\end{array}\right)
$$




$$
=\sum_{j \neq j_{0}} \varphi_{j} d p_{j}+\sum_{l=s+1}^{N} \psi_{l} d \mu_{l}+\sum_{\sigma=1}^{s} \alpha_{\sigma} d \mu_{\sigma}
$$

also vanishes on $U$. So does $\Omega-\frac{1}{2} d_{p, \mu} V$. Since $d p_{j}\left(j \neq j_{0}\right)$ and $d \mu_{l}(s+1$ $\leqq l \leqq N)$ are independent on $U$, coefficients of $d \mu_{l}$ 's must vanish. Hence $\alpha_{l}(s+1 \leqq l \leqq N)$ are rational functions of $\alpha_{1}, \cdots, \alpha_{s}, \mu$ and $p$ on $U$. So are $k_{l}$ 's. But if we consider in a positive $\alpha$ region at generic $(\mu, p) k_{l}$ 's does not depend on $\alpha$, so $k_{l}$ 's are rational functions of $\mu$ and $p$.

Corollary 4.11. Let $G$ be an external graph and $G_{I}$ be its maximal primary subgraph. Then we have the following birational map

$$
L_{G} \boldsymbol{C} \stackrel{\cong}{\longrightarrow} I_{G_{I}}^{\boldsymbol{C}} \times L_{G / I}^{\boldsymbol{C}}
$$

About the codimension formula for a non external graph we have a

Conjecture 4.12. $L_{G \text {, ord }}^{C}$ contains a non singular point of $L_{\widetilde{G}}{ }^{C}$ and

$$
\operatorname{codim} L_{G, \text { ord }}^{\boldsymbol{C}}=\operatorname{codim} L_{\widetilde{G}}^{\boldsymbol{C}}
$$

where $\widetilde{G}$ is an external graph corresponding to $G$. If $G$ is Landauian, $(4 \cdot 8)$ is valid with generic masses fixed.

\section{§ 5. Vacuum Graphs}

As an extreme case of non ordinary components, we consider graphs without external vertices (vacuum graphs) in this section.

The leading Landau variety for a vacuum graph $G$ is defined by

$$
\begin{aligned}
& \Lambda_{G, \text { vac }}^{\boldsymbol{C}}=\text { closure of }\left\{(\mu ; \alpha) \in T^{*} \boldsymbol{C}^{N} \mid \exists \alpha_{l} \neq 0 \quad(l=1, \cdots, N)\right. \\
& \text { and } \exists k_{l} \in \mathbb{C}^{\nu} \text { s.t. } \\
& \text { (I) } \sum_{l=1}^{N}[l: j] k_{l}=0 \quad(j=1, \cdots, n), \\
& \text { (II) } \sum_{l=1}^{N}\left[C_{i}: l\right] \alpha_{l} k_{l}=0 \quad\left(i=1, \cdots, b_{1}(G)\right), \\
& \text { (III) } \left.\mu_{l}+\frac{1}{2} k_{l}{ }^{2}=0 \quad(l=1, \cdots, N)\right\} .
\end{aligned}
$$

$\Lambda_{G \text {,vac }}^{\boldsymbol{C}}$ is homogeneous both in the $\mu_{l}$ 's and the $\alpha_{l}$ 's, so we may also regard the $\alpha_{l}$ 's as base space variables, and the $\mu_{l}$ 's as their dual ones. 
Hereafter the dimensionality $\nu$ in the definition of $\Lambda_{G \text {, vac }}^{C}$ is taken to be sufficiently large.

In terms of the loop momenta $\hat{k}_{i}\left(i=1, \cdots, b_{1}(G)\right)$, the equations (I) and (II) are written in the form

$$
\begin{aligned}
& k_{l}=\sum_{i=1}^{b_{1}(G)}\left[C_{i}: l\right] \hat{k}_{i}, \\
& \left(D \alpha^{t} D\right) \otimes I_{\nu} \cdot\left(\begin{array}{c}
\hat{k}_{1} \\
\vdots \\
\hat{k}_{b_{1}}
\end{array}\right)=0 .
\end{aligned}
$$

Here $D$ denotes the circuit matrix, and we have set

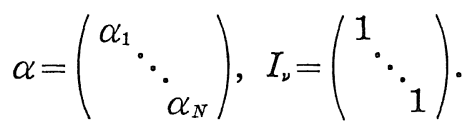

For a fixed value of $\alpha=\alpha^{0}$, let corank $D \alpha^{0 t} D=r$ and take a set of linearly independent solutions $\widehat{K}^{(1)}, \cdots, \widehat{K}^{(r)}$ of the equation $D \alpha^{t} D \cdot \widehat{K}=0$. Then a general solution of $(5 \cdot 2)$ is given by setting

$$
\hat{k}_{i}=\hat{K}_{i}^{(1)} \cdot a^{(1)}+\cdots+\hat{K}_{i}^{(r)} \cdot a^{(r)} \quad\left(i=1, \cdots, b_{1}(G)\right),
$$

where the $a^{(j)}$ 's are arbitrary $\nu$-vectors. This means that the vacuum graph $G$ has an $r$-dimentional realization corresponding to $\alpha=\alpha^{0}$. Now


defines an irreducible component of the variety corank $D \alpha^{t} D=r$ for some $r$. Suppose we have on $L$ a rational parametrization of the form $\alpha_{1}=$ $A_{1}\left(\alpha_{s+1}, \cdots, \alpha_{N}\right), \cdots, \alpha_{s}=A_{s}\left(\alpha_{s+1}, \cdots, \alpha_{N}\right)$. In this case by Lemma 5.1 below we can show that $\Lambda$ is in fact holonomic, and the corresponding potential function is given rationally by

$$
H\left(\mu_{1}, \cdots, \mu_{s}, \alpha_{s+1}, \cdots, \alpha_{N}\right)=\sum_{l=1}^{s} \mu_{l} \cdot A_{l}\left(\alpha_{s+1}, \cdots, \alpha_{N}\right),
$$

so that

$$
\Lambda=\left\{(\alpha ; \mu) \in T^{*} C^{N} \mid \alpha_{l}=\frac{\partial H}{\partial \mu_{l}}(l=1, \cdots, s), \mu_{l^{\prime}}=-\frac{\partial H}{\partial \alpha_{l^{\prime}}}\left(l^{\prime}=s+1, \cdots, N\right)\right\} .
$$


Now we explain the reason why $\Lambda_{G \text {,vac }}^{\boldsymbol{C}}$ is holonomic. Let $\widetilde{G}$ be an external graph corresponding to $G$. $\Lambda_{G \text {,vac }}^{C}$ is obtained by a restriction procedure $p_{j}=0$ from Landau holonomic variety $\Lambda_{\widetilde{G}} \boldsymbol{c}$, hence the defining ideal $J$ of $\Lambda_{G \text {,vac }}^{\boldsymbol{C}}$ satisfies the condition $\{J, J\} \subset J$, where $\{$,$\} means$ the Poisson bracket. Moreover (I), (II), (III) of (5.1) imply

$$
\omega \in J \cdot \Omega_{T^{*} C^{N}}^{1}+\mathcal{O}_{T^{*} C^{N}} \cdot d J
$$

where $\omega$ is the fundamental 1 -form on $T^{*} C^{N}$. Thus the following lemma is sufficient for us.

Lemma 5.1. Let $X$ be a complex manifold and $\pi: T^{*} X \rightarrow X$ be a canonical projection. Let $J \subset \mathcal{O}_{T^{*} X}$ be a coherent Ideal defined by holomorphic functions homogeneous of degree 1 with respect to the fiber of $\pi$ and let $\Lambda=\operatorname{supp} \Theta / J$.

\section{Assume that}

i) $\left.\quad \omega\right|_{\Lambda}=0$

ii) $\{J, J\} \subset J$

iii) $\pi_{*}\left(J \cap \pi^{-1} \mathcal{O}_{X}\right)$ is reduced at a generic point of its support. Then $\Lambda$ is holonomic. Instead of $i)$ if we assume a stronger condition

i)' $\quad \omega \in J \cdot \Omega_{T^{*} X}^{1}+\mathcal{O}_{T^{*} X} \cdot d J$

then $J$ is reduced at a generic point of $\Lambda$.

Proof. Easy.

Before proceeding further, let us explain the motivation of considering such an exceptional case. Firstly, it provides a good example of classification of irreducible components of a given Landau variety. Secondly, the vacuum graphs are considered to play a role in classifying non ordinary components. We give an example of the latter.

Example 5.2. Take a self energy graph $G_{S}$ as in Fig. (4.1).



$G_{s}$ : a self energy graph

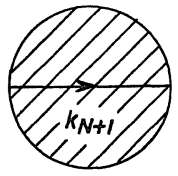

$G_{V}:$ corresponding vacuum graph

Fig. (4.1) 
We consider the associated vacuum graph. Namely joining the two external vertices by a new internal line $N+1$, we get a vacuum graph $G_{V}$. By setting $k_{N+1}=p$, any configuration of $G_{S}$ can be realized as that of $G_{V}$. It is shown that, on the corresponding component $\Lambda$ of $\Lambda_{G_{V}, \text { vac }}^{C} \alpha_{N+1}$ is a rational function of the remaining $\alpha_{l}$ 's, so we may take a local coordinate of the form $\left(\alpha^{\prime}, \mu^{\prime \prime}, \mu_{N+1}\right)$ on $\Lambda$. Let $H_{G_{V}}\left(\alpha^{\prime}, \mu^{\prime \prime}, \mu_{N+1}\right)$ be a potential function for $\Lambda$. Then $\widetilde{H}_{G_{S}}\left(x_{1}, x_{2}, \alpha^{\prime}, \mu^{\prime \prime}\right)=H_{G_{V}}\left(\alpha^{\prime}, \mu^{\prime \prime}\right.$, $\left.-\frac{1}{2} \frac{\left(x_{2}-x_{1}\right)^{2}}{\alpha_{N+1}}\right)$ gives a potential function for $G_{S}$. In this way, classification of irreducible components of a self energy graph is completely reduced to that of a vacuum graph.

For a general graph, some of the components are obtained by considering a vacuum graph where the external vertices are replaced by lines joining each two of them.

In what follows we will decompose the variety defined by corank $D \alpha^{t} D=r$ into irreducible components in the case $r \leqq 2$, and find their rational parametrization.

The case $r=0$ is trivial. In this case $\Lambda=\{\mu=0, \alpha$ :arbitrary $\}$. This component is the ordinary component in $\S 4$.

Before proceeding further we prepare some properties of $U_{G}$. Let $G$ be a connected graph and $D$ be its circuit matrix and $D^{*}$ be its cut set matrix. Then we define

$$
U_{G}(\alpha)=\operatorname{det} D \alpha^{t} D, \alpha=\left(\begin{array}{lll}
\alpha_{1} & & \\
& \ddots & \\
& & \alpha_{N}
\end{array}\right),
$$

and

$$
U_{G}{ }^{*}(\beta)=\operatorname{det} D^{*} \beta^{t} D^{*}, \beta=\left(\begin{array}{lll}
\beta_{1} & & \\
& \ddots & \\
& & \beta_{N}
\end{array}\right) .
$$

The followings are well-known. (Nakanishi [8].)

$$
\begin{aligned}
& U_{G}(\alpha)=\sum_{\left\{l_{1}^{*}, \cdots, l_{b_{1}}{ }^{*}\right\}: \text { cotree }} \alpha_{l_{1}} \cdots \alpha_{l_{b_{1}}}^{*}, \\
& U_{G}^{*}(\beta)=\sum_{\left\{l_{1}, \cdots, l_{\rho}\right\}: \text { tree }} \beta_{l_{1}} \cdots \beta_{l_{\rho}}, \\
& U_{G}^{*}(\beta)=\beta_{1} \cdots \beta_{N} U_{G}\left(\frac{1}{\beta}\right) \\
& U_{G}(\alpha)=\alpha_{1} \cdots \alpha_{N} U_{G}\left(\frac{1}{\alpha}\right)
\end{aligned}
$$


$U_{G}(\alpha)$ and $U_{G}{ }^{*}(\beta)$ are seperately linear in each variable and are sums of monomials of degree $b_{1}(G)$ and $\rho(G)$, respectively.

If we define $U_{G}=U_{G}{ }^{*}=0$ for a non connected graph $G$, we have

$$
\begin{aligned}
& U_{G}(\alpha)=\alpha_{l} U_{G-\{l\}}(\alpha)+U_{G /\{l\}}(\alpha), \\
& U_{G}{ }^{*}(\beta)=\beta_{l} U_{G /\{l\}}^{*}(\beta)+U_{G-\{l\}}^{*}(\beta) .
\end{aligned}
$$

$G$ is called non seperable if it is connected and cannot be seperated into two parts by decomposing it at one vertex.

Proposition 5.2. If $G$ is non seperable, $U_{G}(\alpha)$ is irreducible.

Proof. We prove by an iduction on $N$.

For any line $l$ in $G$, either $G-\{l\}$ or $G /\{l\}$ is non seperable. If $G^{\prime} /\{l\}$ is non seperable, by an induction hypothesis $U_{G /\{l\}}$ is irreducible and so is $U_{G}(\alpha)$ from $(*)$. Therefore assume that for any $l G-\{l\}$ is non seperable and $U_{G-\{l\}}$ is irreducible. If $U_{G}$ is reducible, $U_{G /\{1\}}$ is divisible by $U_{G-\{1\}}$, hence

$$
U_{G}(\alpha)=\left(\alpha_{1}+\cdots+\alpha_{k}\right) U^{\prime}\left(\alpha_{k+1}, \cdots, \alpha_{N}\right)
$$

after re-ordering lines if necessary. By the same argument $U^{\prime}$ contains a linear factor, but $U^{\prime}$ is irreducible. So we have

$$
U_{G}(\alpha)=\left(\alpha_{1}+\cdots+\alpha_{k}\right)\left(\alpha_{k+1}+\cdots+\alpha_{N}\right) .
$$

This implies that $G$ is a union of two simple loops which is a contradiction with the assumption that $G$ is non seperable.

Now we can give the results for the case $r=1$.

Theorem 5.3. When $r=1 L$ is defined by $U_{G}(\alpha)=0$. If $G$ is non seperable, $U_{G}(\alpha)$ is irreducible and there is only one component

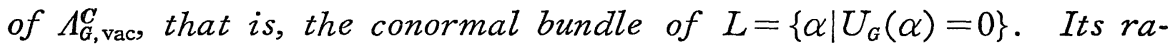
tional parametrization is given by

$$
\alpha_{l}=-\frac{U_{G /\{l\}}}{U_{G-\{l\}}}
$$

for any $l$. 
Now we consider the case $r=2$. Since we are dealing with a leading singularity, we may use $\beta_{l}=1 / \alpha_{l}$ and $D^{*} \beta^{t} D^{*}$ instead of $\alpha_{l}$ and $D \alpha^{t} D$. We assume that $G$ is connected and $\rho(G) \geqq 2$. We fix a reference tree $T$, then we have a unique cut set matrix $D^{*}$ (Nakanishi [8], Theorem 2-29). We denote by $U^{*}\left(\begin{array}{l}a_{1} \cdots a_{r} \\ b_{1} \cdots b_{r}\end{array}\right)$ a signed minor of $D^{*} \beta^{t} D^{*}$. Note that this is dependent on the choice of $T$. If $a, b, c \in T$, we have $D^{*} \beta^{t} D^{*}$ in the following form:

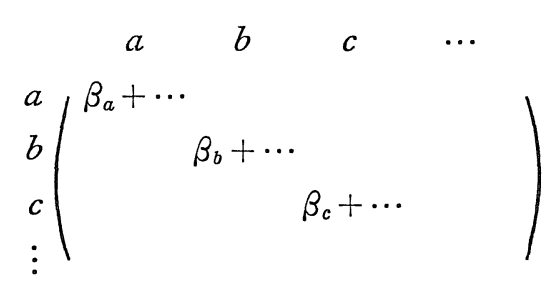

where $\beta_{a}, \beta_{b}, \beta_{c}$ appear only in the places explicitly written here.

Lemma 5.4. $U\left(\begin{array}{c}a_{1} \cdots a_{r} \\ b_{1} \cdots b_{r}\end{array}\right)=\sum \pm \alpha_{l_{1}} \cdots \alpha_{l_{b_{1}}-r}$

where the sum is taken over $\left\{l_{1}, \cdots, l_{b_{1}-r}\right\}$ that makes a cotree in both $G-\left\{a_{1}, \cdots, a_{r}\right\}$ and $G-\left\{b_{1}, \cdots, b_{r}\right\}$.

$$
U^{*}\left(\begin{array}{l}
a_{1} \cdots a_{r} \\
b_{1} \cdots b_{r}
\end{array}\right)=\sum \pm \beta_{l_{1}} \cdots \beta_{l \rho-r}
$$

where the sum is taken over $\left\{l_{1}, \cdots, l_{\rho-r}\right\}$ that makes a tree in both $G /\left\{a_{1}, \cdots, a_{r}\right\}$ and $G /\left\{b_{1}, \cdots, b_{r}\right\}$.

Proof. We give a proof for $U\left(\begin{array}{l}a \\ b\end{array}\right)$. Other cases can be proved similarly.

Expanding the determinant we have

$$
\begin{aligned}
U\left(\begin{array}{l}
a \\
b
\end{array}\right)= & \sum \alpha_{l_{1}} \cdots \alpha_{l_{b_{1}-1}}\left|\begin{array}{c}
{\left[C_{b}: l_{1}\right] \cdots\left[C_{b}: l_{b_{1}-1}\right]} \\
{\left[C_{c}: l_{1}\right] \cdots\left[C_{c}: l_{b_{1}-1}\right]} \\
\vdots \\
\vdots
\end{array}\right| \\
& \times\left|\begin{array}{c}
{\left[C_{a}: l_{1}\right] \cdots\left[C_{a}: l_{b_{1}-1}\right]} \\
{\left[C_{c}: l_{1}\right] \cdots\left[C_{c}: l_{b_{1}-1}\right]} \\
\vdots
\end{array}\right|
\end{aligned}
$$


In general for a graph $G$ and independent set of loops $\left\{C_{1}, \cdots, C_{b_{1}}\right\}$ it is well known that

$$
\left|\begin{array}{cc}
{\left[C_{1}: l_{1}\right]} & \cdots\left[C_{1}: l_{b_{1}}\right] \\
\vdots & \vdots \\
{\left[C_{b_{1}}: l_{1}\right]} & \cdots\left[C_{b_{1}}: l_{b_{1}}\right]
\end{array}\right|=\left\{\begin{array}{cc} 
\pm 1 & \text { if }\left\{l_{1}, \cdots, l_{b_{1}}\right\} \text { is a cotree } \\
0 & \text { otherwise. }
\end{array}\right.
$$

Hence the proposition follows.

Proposition 5.5. $U_{G} *\left(\begin{array}{l}a \\ b\end{array}\right) \not \equiv 0$ if and only if $a$ and $b$ belong to the same non seperable component.

Proof. The necessity is trivial. To prove the sufficiency by Lemma 5.4 it is sufficient to show the existence of a set $\left\{l_{1}, \cdots, l_{p-1}\right\}$ such that $\left\{l_{1}, \cdots, l_{\rho-1}, a\right\}$ and $\left\{l_{1}, \cdots, l_{\rho-1}, b\right\}$ are both trees in $G$. If $\{a, b\}$ is a cut set, we may take a tree in each connected components of $G-\{a, b\}$ and make a union as $\left\{l_{1}, \cdots, l_{\rho-1}\right\}$.

Otherwise there exists a cortree containing $a$ and $b$. Then we have the following

Lemma 5.6. If $a, b$ belong to the same cotree and the same non seperable component, there exist two loops $C$ and $C^{\prime}$ such that

$$
C \ni a, C \nexists b ; \quad C^{\prime} \nexists a, C^{\prime} \ni b ; \quad C \cap C^{\prime} \neq \phi .
$$

We omit the proof of this lemma.

End of the proof of Proposition 5. 5. Take a line $l_{0}$ in $C \cap C^{\prime}$. If we choose a tree $\left\{l_{1}, \cdots, l_{\rho-1}\right\}$ in $(G-\{a, b\}) /\left\{l_{0}\right\}$, this satisfies the above conditions.

Definition 5.7. A graph $G$ is k-connected if and only if any two distinct points can be connected by at least $k$ paths each two of which have no common point other than two end points.

Note that if $G$ is $k$ connected, for any line $l G-\{l\}$ and $G /\{l\}$ are $k-1$ connected and at least one of them is $k$ connected. 
Proposition 5.8. If $G$ is 3-connected, $U_{G} *\left(\begin{array}{l}a \\ b\end{array}\right)$ is a non zero irreducible polynomial.

Proof. We may assume that $G$ has no self loop. If $\rho(G)=2$ or 3 , we can prove the proposition directly. If for some $l \in T-\{a, b\} G-\{l\}$ is 3 connected, by the induction hypothesis $U_{G-\{l\}}^{*}\left(\begin{array}{l}a \\ b\end{array}\right)$ is irreducible. So

$$
U_{G}^{*}\left(\begin{array}{c}
a \\
b
\end{array}\right)=\beta_{l} U_{G /\{l\}}^{*}\left(\begin{array}{c}
a \\
b
\end{array}\right)+U_{G-\{l\}}^{*}\left(\begin{array}{c}
a \\
b
\end{array}\right)
$$

is also irreducible. Now we may assume that $G-\{l\}$ is 2 connected and $G /\{l\}$ is 3-connected for any $l \in T-\{a, b\}$. As $\rho(G) \geqq 4$ we can take distinct $l_{1}, l_{2} \in T-\{a, b\}$. By Proposition $5.5 U_{G /\left\{l_{i}\right\}}^{*}\left(\begin{array}{l}a \\ b\end{array}\right) \neq 0$ and by the induction hypothesis $U_{G /\left\{l_{i}\right\}}^{*}\left(\begin{array}{l}a \\ b\end{array}\right)$ is irreducible, hence if $U_{G}^{*}\left(\begin{array}{l}a \\ b\end{array}\right)$ is reducible we have

$$
\begin{aligned}
U_{G}^{*}\left(\begin{array}{l}
a \\
b
\end{array}\right) & =\left(\beta_{l_{1}}+\cdots\right) U_{G /\left\{l_{1}\right\}}^{*}\left(\begin{array}{l}
a \\
b
\end{array}\right) \\
& =\left(\beta_{l_{2}}+\cdots\right) U_{G /\left\{l_{2}\right\}}^{*}\left(\begin{array}{l}
a \\
b
\end{array}\right) .
\end{aligned}
$$

Hence $U_{G} *\left(\begin{array}{l}a \\ b\end{array}\right)=\left(\beta_{l_{1}}+\cdots\right)\left(\beta_{l_{2}}+\cdots\right)$. This implies $\rho(G)=3$ which is a contradiction.

Theorem 5.9. Let $G$ be a 3-connected graph with no multiple lines. Then the variety defined by corank $D^{*} \beta^{t} D^{*}=2$ is an irreducible variety of codimensions 3 . If $l_{1}, l_{2}, l_{3}$ belong to a tree, $\beta_{1}, \beta_{2}, \beta_{3}$ can be expressed as rational functions of other $\beta_{l}$ 's.

Proof. In general for a symmetric matrix $A$ the variety defined by corank $A \leqq r$ is of codimensions less than $\frac{r(r+1)}{2}$. Thus in our case the codimension is less than 3 .

First we prove that for $a, b \in T$ there exists a unique irreducible component $L$ such that $\left.U_{G /\{a, b\}}^{*}\right|_{L} \not \equiv 0$. From

$$
0=U_{G}^{*}\left(\begin{array}{l}
b \\
b
\end{array}\right)=U_{G /\{b\}}^{*}=\beta_{a} U_{G /\{a, b\}}^{*}+U_{(G-\{a\}) /\{b\}}^{*}
$$


we have $\beta_{a}=-U_{(G-\{a\}) /\{b\}}^{*} / U_{G /\{a, b\}}^{*}$.

Note that from the form of the matrix $(* *)$ the right hand side involves neither $\beta_{a}$ nor $\beta_{b}$. $\beta_{b}$ can be expressed likewise. If we choose $c \in T$ $-\{a, b\}$ we have

$$
0=U_{G}^{*}\left(\begin{array}{l}
a \\
b
\end{array}\right)=\beta_{c} U_{G /\{c\}}^{*}\left(\begin{array}{l}
a \\
b
\end{array}\right)+U_{G-\{c\}}^{*}\left(\begin{array}{l}
a \\
b
\end{array}\right)
$$

From Proposition 5.5 and the assumption on $G, U_{G /\{c\}}^{*}\left(\begin{array}{l}a \\ b\end{array}\right) \neq 0$ and $U_{G-\{c\}}^{*}\left(\begin{array}{l}a \\ b\end{array}\right)$ $\not \equiv 0$. Thus $\beta_{c}$ can be expressed as a rational function of $\beta_{l}$ 's other than $\beta_{a}, \beta_{b}, \beta_{c}$.

Since

$$
(* * *) \quad U_{G /\{a, b\}}^{*}=U_{G /\{c\}}^{*}\left(\begin{array}{l}
a \\
b
\end{array}\right)^{-1} U_{G /\{a\}}^{*}\left(\begin{array}{l}
b \\
c
\end{array}\right) U_{G /\{b\}}^{*}\left(\begin{array}{l}
a \\
c
\end{array}\right)
$$

we can substitute $\beta_{c}$ in the expression of $\beta_{a}$ and $\beta_{b}$. Thus we have an irreducible component $L$ of codimensions 3. By Proposition 5.8, $U_{G} *\left(\begin{array}{l}a \\ b\end{array}\right)$ is irreducible, hence the above component does not depend on the choice of $c$. The expression for $\beta_{a}\left(\beta_{b}\right)$ thus obtained coincides with that obtained from $U_{G} *\left(\begin{array}{l}b \\ c\end{array}\right)=0\left(U_{G}^{*}\left(\begin{array}{l}a \\ c\end{array}\right)=0\right)$, hence it is not identically zero.

Thus we have a unique component $L$ such that $\left.U_{G /\{a, b\}}^{*}\right|_{L} \neq 0$. Moreover from $(* * *)$ we see that $U_{G /\{b, c\}}^{*} \not \equiv 0$, hence the component obtained by the above method is unique. Since $r=2$, on any component $U_{G /\{a, b\}}^{*} \neq 0$ for some $a, b$. Thus we have proved the component is unique.

For a general graph $G$, consider a mapping $\varphi$ of internal lines to another graph $G_{1}$, such that for each line $l_{0}$ in $G_{1}, \varphi^{-1}\left(l_{0}\right)$ is a self energy part (that is, $G_{l_{0}}=\varphi^{-1}\left(l_{0}\right)$ is connected and adjacent to the rest of $G$ by two vertices a, b). This map induces in $\alpha$-space the following rational map $\widetilde{\varphi}:\left(\alpha_{1}, \cdots, \alpha_{n}\right) \mapsto\left(\alpha_{1}^{0}, \cdots, \alpha_{N_{0}}^{0}\right)$, where

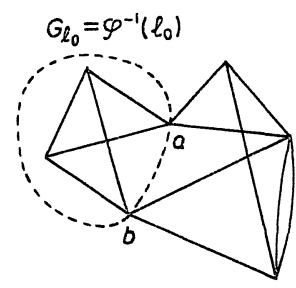

G



Fig. (4.2)

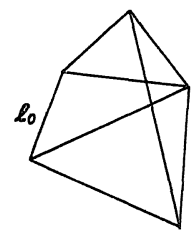

$G_{1}$ 


$$
\alpha_{l_{0}}^{0}=\frac{U_{G_{l_{0}[\{a, b\}]}}(\alpha)}{U_{G_{l_{0}}}(\alpha)} .
$$

Corresponding to each $r$-dimensional irreducible component of $\Lambda_{G \text {,vac }}^{C}$, its proper transform by $\widetilde{\varphi}$ defines an $r$-dimensional component of $\Lambda_{G \text {, vac }}^{\boldsymbol{C}}$ on which each $G_{l_{0}}$ has a 1-dimensional realization. In particular, if $r=2$ and $G_{1}$ is 3 -connected with no multiple lines, the corresponding component of $G$ has a rational parametrization of codimensions 3 . But this is not quite sufficient as illustrated by the following example.

Example. 5.9 .

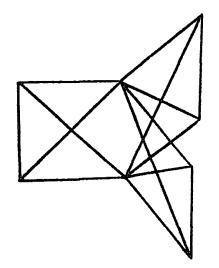

Fig. (4.3)

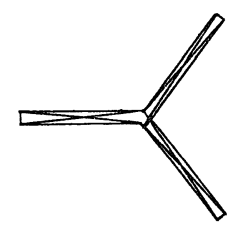

(a)

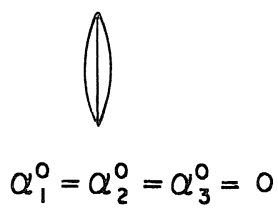

(A)

For the graph of Figure (4.3), its configuration (a) is obtained by taking the triple line graph $(\mathrm{A})$ as $G_{1}$. Similarly the configuration (b) of Fig. (4.4) is obtained by considering the non 3-connected graph (B).

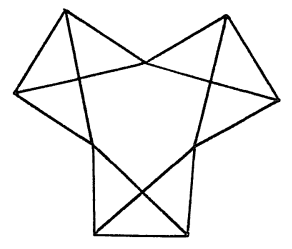

Fig. (4.4)



(b)

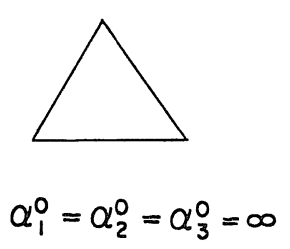

(B)

We conjecture that the above procedure gives all the components with $r=2$, if we admit as $G_{1}$ the two exceptional graphs (A) and (B) in addition.

\section{§ 6. Feynman Integrals}

The Feynman integral $F_{G, \sigma}(p)$ corresponding to a Feynman graph $\left(G, J_{0}, \sigma\right)$ is defined by the formula 


$$
\begin{aligned}
F_{G, \sigma}(p)= & \int \cdots \int_{\boldsymbol{R}^{\nu N}} \prod_{j=1}^{n}(2 \pi)^{\nu} \delta\left(\sum_{i \in \sigma^{-1}(j)} p_{i}-\sum_{l \in I_{0}}[l: j] k_{l}\right) \\
& \times \prod_{l \in I_{0}} \frac{i}{{k_{l}}^{2}-m_{l}{ }^{2}+i 0} \cdot \frac{d k_{l}}{(2 \pi)^{\nu}},
\end{aligned}
$$

although mathematically it is not a meaningful expression in two respects; (i) the fibre of integration is not compact (ultraviolet divergence), and (ii) the product in the integrand $\Phi_{G, \sigma}$ is not well defind in general if some of the $m_{l}$ 's vanish (infrared divergence). So we begin by giving the rigorous definition of $(6 \cdot 1)$ through a compactification of integration space $\boldsymbol{R}^{\nu N}$ (cf. Boyling [1], Fotiadi [4]). Following Speer [13] we introduce generalized Feynman integrals with complex parameters $\lambda_{l}$ 's:

$$
\begin{aligned}
F_{G, \sigma}(p ; \lambda)=\int & \cdots \int_{\boldsymbol{R}^{\nu N}} \prod_{j=1}^{n}(2 \pi)^{\nu} \delta\left(\sum_{i \in \sigma^{-1}(j)} p_{i}-\sum[l: j] k_{l}\right) \\
& \times i\left(k_{l}{ }^{2}-m_{l}{ }^{2}+i 0\right)^{-\lambda_{l}} \frac{d k_{l}}{(2 \pi)^{\nu}} .
\end{aligned}
$$

Throughout this section we may assume that $G$ is connected with no loss of generality.

Let $X=\left(\boldsymbol{P}^{\nu}\right)^{N}$ be a product of $N$ copies of a $\nu$-dimensional real projective space. We denote by $\left(k_{l}, s_{l}\right)_{l \in I_{0}}$ its homogeneous coordinate, and set $X^{\prime}=\left(\boldsymbol{P}^{\nu}\right)^{N}-\bigcup_{l \in I_{0}}\left\{s_{l}=0\right\}-\underset{l \in I_{0}-I_{M}}{\bigcup}\left\{k_{l}=0\right\}$, where $I_{M}=\left\{l \in I_{0} \mid m_{l} \neq 0\right\}$ is the set of massive internal lines. Let $\widetilde{X}$ be the monoidal transform of $X$ along the ideal $\prod_{I \subset I_{0}}\left(s_{l}\right)_{l \in I} \cdot \prod_{I^{\prime} \subset I_{0}-I_{M}}\left(k_{l 0}, \cdots, k_{l \nu-1}\right)_{l \in I^{\prime}}$, where $\left(s_{l}\right)_{l \in I}\left(\right.$ resp. $\left(k_{l 0} \ldots, k_{l \nu-1}\right)$ $l_{l \in I^{\prime}}$ ) denotes the homogeneous ideal generated by $\left\{s_{l} \mid l \in I\right\}$ (resp. $\left\{k_{l \mu} \mid l\right.$ $\left.\left.\in I^{\prime}, \mu=0, \cdots, \nu-1\right\}\right) . \quad \widetilde{X}$ is a compact algebraic manifold. We also introduce

$$
\begin{aligned}
& V= \text { the closure in } \boldsymbol{R}^{\nu n_{0}} \times X \text { of }\left\{(p, k, s) \in \boldsymbol{R}^{\nu n_{0}} \times X^{\prime} \mid\right. \\
&\left.\sum_{i \in \sigma^{-1}(j)} p_{i}-\sum_{l \in I_{0}}[l: j] k_{l} / s_{l}=0 \quad(j=1, \cdots, n)\right\}, \\
& S_{I, I^{\prime}}=\left\{(p, k, s) \in \boldsymbol{R}^{\nu n_{0}} \times X \mid s_{l}=0(l \in I), k_{l^{\prime}}=0\left(l^{\prime} \in I^{\prime}\right)\right\},
\end{aligned}
$$

and denote by $\widetilde{X}\left(\right.$ resp. $\left.\widetilde{S}_{I, I^{\prime}}\right)$ the set $\overline{\pi^{-1}\left(V \cap\left(\boldsymbol{R}^{\nu n_{0}} \times X^{\prime}\right)\right.}$ ) (resp. $\left.\overline{\pi^{-1}\left(S_{I, I^{\prime}} \cap\left(\boldsymbol{R}^{\nu n_{0}} \times X\right)\right)}\right)$, where $\pi: \boldsymbol{R}^{\nu n_{0}} \times \widetilde{X} \rightarrow \boldsymbol{R}^{\nu n_{0}} \times X$ is the projection.

Proposition 6.1. $\widetilde{V}$ is non singular. 
Proof. Take a point $\widetilde{P}_{0} \in \widetilde{V}$, and set $I^{i}=\left\{l \in I_{0}-I_{M} \mid k_{l}{ }^{0}=0\right\}, I^{u}=\{l \in$ $\left.I_{0} \mid s_{l}{ }^{0}=0\right\}$, where $\left(p^{0}, k^{0}, s^{0}\right)=\pi\left(\widetilde{P}_{0}\right)$ denotes the projection of $\widetilde{P}_{0}$. Clearly $I^{i} \cap I^{u}=\phi$. Let $G^{\infty}$ be a graph obtained by adding a new vertex $\infty$ to $G$ and joining all the external lines to $\infty$. We take an ordering of internal lines of $G^{\infty}$ so that

$$
\begin{aligned}
& I_{1}=I^{i}=\left\{1, \cdots, N_{1}\right\}, \\
& I_{2}=I_{0}-I^{i} \sqcup I^{u}=\left\{N_{1}+1, \cdots, N_{2}\right\}, \\
& I_{3}=\{\text { external lines of } G\}=\left\{N_{2}+1, \cdots, N_{3}\right\}, \\
& I_{4}=I^{u}=\left\{N_{3}+1, \cdots, N\right\}, \\
& I_{0}^{\infty}=I_{1} \sqcup I_{2} \sqcup I_{3} \sqcup I_{4} .
\end{aligned}
$$

By reordering lines if necessary, we take a homogeneous local coordinate $(\bar{k}, t)$ of $\widetilde{X}$ such that

$$
\begin{aligned}
& k_{l_{1}}=t_{l_{1}} \cdots t_{N_{1}} \bar{k}_{l_{1}}, s_{l_{1}}=1 \quad\left(l_{1} \in I_{1}\right), \\
& k_{l_{2}}=\bar{k}_{l_{2}}, s_{l_{2}}=t_{l_{2}}\left(l_{2} \in I_{2}\right), \\
& k_{l_{4}}=\bar{k}_{l_{4}}, s_{l_{4}}=t_{N_{3}+1} \cdots t_{l_{4}}\left(l_{4} \in I_{4}\right), \\
& \bar{k}_{l} \neq 0 \text { at } \widetilde{P}_{0}\left(l \in I_{0}-I_{M} \text { or } l_{4} \in I_{4}\right) .
\end{aligned}
$$

Finally take a tree $T$ in $G^{\infty}$ by choosing lines successively from $N$ to 1 in the inverse order, and set $T^{*}=I_{0}^{\infty}-T, T_{j}=T \cap I_{j}, T_{j}{ }^{*}=T^{*} \cap I_{j}$ $(j=1, \cdots, 4)$. Then, in a neighbourhood of $\widetilde{P}_{0}, \widetilde{V}$ is defined by the following equations:

$$
\begin{aligned}
& \bar{k}_{l_{1}}=\sum_{l_{1} * T_{1} *}\left[C_{l_{1}}: l_{1}\right] t_{l_{1} * \cdots} t_{l_{1}-1} \bar{k}_{l_{1} *} \\
& \left(l_{1} \in T_{1}\right) \text {, } \\
& \bar{k}_{l_{2}}=\sum_{l_{1} * T_{1}^{*}}\left[C_{l_{1}^{*}}: l_{2}\right] t_{l_{1^{*}} \cdots t_{N_{1}}} \bar{k}_{l_{1^{*}}}+\sum_{l_{2^{*} \in T_{2}^{*}}}\left[C_{l_{2^{*}}}: l_{2}\right] \bar{k}_{l_{2^{*}}} \quad\left(l_{2} \in T_{2}\right) \text {, } \\
& p_{l_{3}}=\sum_{l_{1}^{*} \in T_{1^{*}}}\left[C_{l_{1}^{*}}: l_{3}\right] t_{l_{1^{*}} \cdots t_{N_{1}}} \bar{k}_{l_{1^{*}}}+\sum_{l_{2^{*} \in T_{2}}}\left[C_{l_{2^{*}}}: l_{3}\right] \bar{k}_{l_{2^{*}}} \\
& +\sum_{l_{3^{*} \in T_{3}^{*}}}\left[C_{l_{3^{*}}} ; l_{3}\right] p_{l_{3^{*}}} \\
& \left(l_{3} \in T_{3}\right), \\
& \bar{k}_{l_{4}}=\left(\sum_{l_{1} * T_{1}^{*}}\left[C_{l_{1}}: l_{4}\right] t_{l_{1} *} \cdots t_{N 1_{1}} \bar{k}_{l_{1}^{*}}+\sum_{l_{2} * \in T_{2}{ }^{*}}\left[C_{l_{2}}: l_{4}\right] \bar{k}_{l_{2^{*}}}+\sum_{l_{3} * T_{3}{ }^{*}}\left[C_{l_{3}^{*}}: l_{4}\right] p_{l_{3}}\right) \cdot t_{N_{3}+1} \cdots t_{l_{4}} \\
& +\sum_{l_{4}^{*} \in T_{4}^{*}}\left[C_{l_{4}^{*}}: l_{4}\right] t_{l_{4^{*}+1}} \cdots t_{l_{4}} \bar{k}_{l_{4^{*}}} \\
& \left(l_{4} \in T_{4}\right) .
\end{aligned}
$$


Here $C_{l^{*}}$ denotes the circuit in $G^{\infty}$ corresponding to $l^{*} \in T^{*}$. Note that $\left[C_{l^{*}}: l\right] \neq 0$ implies $l^{*}<l$ by the choice of $T$. From $(6 \cdot 3)$ the proposition is obvious.

Remark. Because $\bar{k}_{l} \neq 0$ for $l \in\left(I_{0}-I_{M}\right) \sqcup I_{4}$ in (6.3), some of the $t_{l}$ 's cannot vanish at $\widetilde{P}_{0}$. Therefore we may take an inhomogeneous coordinate system $\left(k^{\prime}, t^{i}, t^{u}\right)$ in the following way:

$$
\begin{array}{ll}
k_{l_{1}}=\prod_{l_{1} \in I_{\rho}^{i}} t_{\rho}^{i} k_{l_{1}}^{\prime}, s_{l_{1}}=1 & \left(l_{1} \in I^{i}\right), \\
k_{l_{4}}=k_{l_{4}}^{\prime}, s_{l_{4}}=\prod_{l_{4} \in I_{\rho^{\prime}}^{u}} t_{\rho^{\prime}}^{u} & \left(l_{4} \in I^{u}\right), \\
k_{l_{2}}=k_{l_{2}}^{\prime}, s_{l_{2}}=1 & \left(l_{2} \in I_{2}\right) .
\end{array}
$$

Here $I^{i}=I_{r}^{i} \supsetneqq I_{r-1}^{i} \supsetneqq \cdots \supsetneqq I_{1}^{i} \supsetneqq I_{0}^{i}=\phi$ and $I^{u}=I_{1}^{u} \supsetneqq I_{2}^{u} \supsetneqq \cdots \supsetneqq I_{r^{\prime}}^{u} \supsetneqq I_{r^{\prime}+1}^{u}=\phi$ are chains consisting of subsets of internal lines, and if $l=\max _{l^{\prime} \in I_{\rho}^{i}} l^{\prime}$ or $\min _{l^{\prime} \in I_{\rho}^{u}} l^{\prime}$, then one of the non zero components of $k_{l}{ }^{\prime}$ is normalized to 1 .

Theorem 6.2. If $2 \sum_{l \in I} \lambda_{l} \notin Z$ for any $I \subset I_{0}$, the integrand of (6-2) has an extension as a hyperfunction density on $\boldsymbol{R}^{\nu n_{0}} \times \widetilde{X}$. Integral $(6 \cdot 2)$ is then well defined, and

$$
S S F_{G, \sigma}(p ; \lambda) \subset \underset{\substack{I \subset I_{0}-I^{\prime} \\ I^{\prime} \subset I_{0}-I_{M G}}}{\bigcup} \Lambda_{\left(G-I^{\prime}\right) / I, \tau_{I} \sigma}^{+}
$$

holds.

Proof. In the coordinate of the above remark, the integrand density of $(6 \cdot 2)$ is written in the following form:

$$
\begin{gathered}
\Phi_{G, \sigma}=i^{N}(2 \pi)^{\nu(n-N)} \delta_{\widetilde{v}} \prod_{l \in I_{0}}\left(k_{l}{ }^{2}-s_{l}(t)^{2} m_{l}{ }^{2}+i 0\right)^{-\lambda_{l}} \prod_{\rho=1}^{r}\left|t_{\rho}^{i}\right|^{\lambda_{\rho}^{i}} \prod_{\rho^{\prime}=1}^{r^{\prime}}\left|t_{\rho^{\prime}}^{u}\right|^{\lambda_{\rho^{\prime}}^{u}} \\
\cdot \prod_{i \in I_{0}}\left|d k_{l}\right| \prod_{\rho=1}^{r}\left|d t_{\rho}^{i}\right| \prod_{\rho^{\prime}=1}^{r^{\prime}}\left|d t_{\rho^{i}}^{u}\right|
\end{gathered}
$$

where

$$
\begin{array}{ll}
\delta \widetilde{v}=\prod_{l \in I_{0} \cap T} \delta\left(k_{l}-f_{l}\right) \prod_{l_{3} \in T_{3}} \delta\left(p_{l_{3}}-f_{l_{3}}\right), & \\
f_{l_{1}}=\sum_{l_{1} * \in T_{1} *}\left[C_{l_{1}^{*}}: l_{1}\right]\left(s_{l_{1}^{*}}(t) / s_{l_{1}}(t)\right) k_{l_{1} *} & \left(l_{l} \in T_{1}\right), \\
f_{l_{2}}=\sum_{l_{1^{*} \in T_{1} *}}\left[C_{l_{1^{*}}}: l_{2}\right] k_{l_{1^{*}}}+\sum_{l_{2} * T_{2^{*}}}\left[C_{l_{2^{*}}:}: l_{2}\right] k_{l_{2^{*}}} & \left(l_{2} \in T_{2}\right),
\end{array}
$$




$$
\begin{aligned}
& f_{l_{3}}=\sum_{l_{1} * T_{1} *}\left[C_{l_{1}^{*}}: l_{3}\right] s_{l_{1^{*}}}(t) k_{l_{1} *}+\sum_{l_{2} * T_{2^{*}}}\left[C_{l_{2}^{*}}: l_{3}\right] k_{l_{2^{*}}} \\
& +\sum_{l_{3} * T_{3}^{*}}\left[C_{l_{3}^{*}}: l_{3}\right] p_{l_{3}^{*}} \quad\left(l_{3} \in T_{3}\right) \\
& f_{l_{4}}=\left(\sum_{l_{1}^{*} \in T_{1}^{*}}\left[C_{l_{1}}: l_{4}\right] s_{l_{1}^{*}}(t) k_{l_{1^{*}}}+\sum_{l_{2^{*} \in T_{2}}}\left[C_{l_{2^{*}}}: l_{4}\right] k_{l_{2^{*}}}+\sum_{l_{3^{*} \in T_{3}^{*}}}\left[C_{l_{3^{*}}}: l_{4}\right] p_{l_{3}^{*}}\right) \cdot s_{l_{4}}(t) \\
& +\sum_{l_{4}^{*} \in T_{4}^{*}}\left[C_{l_{4}^{*}}: l_{4}\right]\left(s_{l_{4}}(t) / s_{l_{4^{*}}}(t)\right) k_{l_{4^{*}}} \quad\left(l_{4} \in T_{4}\right), \\
& s_{l_{1}}(t)=\prod_{l_{1} \in I_{\rho}^{i}} t_{\rho}^{i} \quad\left(l_{1} \in I^{i}\right), \\
& s_{l_{2}}(t)=1 \quad\left(l_{2} \in I_{2}\right), \\
& s_{l_{4}}(t)=\prod_{l_{\sharp} \in I_{\rho^{\prime}}^{u}} t_{\rho^{\prime}}^{u} \quad\left(l_{4} \in I^{u}\right) .
\end{aligned}
$$

We have omitted primes for $k_{l}$ 's under the convention that the normalized components are to be dropped from $d k_{l}$. The exponents of $\left|t_{\rho}^{i}\right|,\left|t_{\rho^{\prime}}^{u}\right|$ are given by the formulae

$$
\begin{aligned}
& \lambda_{\rho}^{i}=-2 \sum_{l \in I_{\rho}^{i}} \lambda_{l}+\nu b_{1}\left(Q_{I_{\rho}^{i}}\right)-1, \\
& \lambda_{\rho^{\prime}}^{u}=2 \sum_{l \in I_{\rho^{\prime}}^{u}} \lambda_{l}-\nu b_{1}\left(G_{I_{\rho}^{u}}\right)-1,
\end{aligned}
$$

where $Q_{I_{\rho}^{i}}$ denotes the graph obtained by identifying all the external vertices of $G_{I_{\rho}^{i} \text {. }} \quad$ In particular $\left|t_{\rho}^{i}\right|^{\lambda_{\rho}^{i}}$ and $\left|t_{\rho^{\prime}}^{u}\right|^{\lambda_{\rho^{\prime}}^{u}}$ are well defind if $2 \sum_{l \in I} \lambda_{l} \notin \mathbb{Z}$ for any $I \subset I_{0}$. We now show that the product is well defined at $\widetilde{P}_{0}$. To see this, it is sufficient to show that $\sum_{l \in T} v_{l} . d\left(k_{l}-f_{l}\right)+(1 / 2) \sum_{l \in I_{0}} \alpha_{l} d\left(k_{l}{ }^{2}-\right.$ $\left.s_{l}(t)^{2} m_{l}{ }^{2}\right)+\sum_{\rho=1}^{r} \tau_{\rho}^{i} d t_{\rho}^{i}+\sum_{\rho^{\prime}=1}^{r^{\prime}} \tau_{\rho^{\prime}}^{u} d t_{\rho^{\prime}}^{u}=0$ at $\widetilde{P}_{0}$ and $\alpha_{l}\left(k_{l}{ }^{2}-s_{l}(t)^{2} m_{l}{ }^{2}\right)=0$ with $\alpha_{l}$ $\geqq 0 \quad\left(l \in I_{0}\right)$ implies $v_{l}=0, \alpha_{l}=\tau_{\rho}^{i}=\tau_{\rho^{\prime}}^{u}=0$. Consider first the subgraph $G_{I_{r}^{u}}$. From the condition above, we have $\sum_{l \in I_{r}^{u}}[C: l] \alpha_{l} k_{l}=0$ and $\sum_{l \in I_{r}^{u}}[l: j] k_{l}$ $=0$ for any circuit $C$ and any vertex $j$ of $G_{I_{r}^{u}}^{u}$. Since $k_{l} \neq 0$ and $\alpha_{l} \geqq 0$, this is possible only when $\alpha_{l}=0\left(l \in I_{r^{\prime}}^{u}\right)$ and $v_{l}=0\left(l \in I_{r^{\prime}}^{u} \cap T\right)$. Repeating the same argument we see inductively that $\alpha_{l}=0 \quad\left(l \in I_{0}\right), v_{l}=0$ $(l \in T)$, hence $\tau_{\rho}^{i}=\tau_{\rho^{\prime}}^{u}=0$. Thus $\Phi_{G, \sigma}$ admits an extension to $\boldsymbol{R}^{\nu n_{0}} \times \widetilde{X}$. Since $\widetilde{X}$ is compact, integration (6.2) now makes sense and we can estimate the singularity spectrum of $F_{G, \sigma}$ by applying the theory of integration of hyperfunctions [11]. From (6.5) we see that the contribution from $S S \Phi_{G, \sigma} \cap \widetilde{S}_{I^{i}, I^{u}}$ to $S S F_{G, \sigma}$ is confined to the following set: 


$$
\begin{aligned}
& \left\{(p ; x) \in T^{*} \boldsymbol{R}^{\nu n_{0}} \mid \exists k_{l_{2}} \in \boldsymbol{R}^{\nu},{ }^{\exists} \alpha_{l_{2}} \geqq 0\left(l_{2} \in I_{2}\right)\right. \text { such that } \\
& \sum_{l_{3} \in I_{3}}\left[C_{l_{3} *}: l_{3}\right] x_{l_{3}}=0, \\
& \sum_{l_{3} \in T_{3}}\left[C_{l_{2^{*}}}: l_{3}\right] x_{l_{2}}=\sum_{l_{2} \in I_{2}}\left[C_{l_{2}^{*}}: l_{2}\right] \alpha_{l_{2}} k_{l_{2}}, \\
& \alpha_{l_{2}}\left(k_{l_{2}}^{2}-m_{l_{2}}^{2}\right)=0 \\
& \left(l_{2} \in I_{2}\right) \text {, } \\
& k_{l_{2}}=\sum_{l_{2^{*} \in T_{2}}}\left[C_{l_{2} *}: l_{2}\right] k_{l_{2^{*}}} \quad\left(l_{2} \in T_{2}\right) \text {, } \\
& \left.p_{l_{3}}=\sum_{l_{2^{*} \in T_{2^{*}}}}\left[C_{l_{2^{*}}}: l_{3}\right] k_{l_{2^{*}}}+\sum_{l_{3^{*} \in T_{3}}}\left[C_{l_{3} *}: l_{3}\right] p_{l_{3^{*}}}\left(l_{3} \in T_{3}\right)\right\} \text {. }
\end{aligned}
$$

It is easy to show that $(6 \cdot 7)$ is contained in $\bigcup_{I_{0}-I^{i} \supset I \supset I^{u}} \Lambda_{\left(G-I^{i}\right) / I, \tau_{I} \sigma}^{+}$This completes the proof of the theorem.

Remark. It is known that a function of the form (6.5) is a solution of a holonomic system. See Kashiwara-Kawai [7], Theorem 1.

The $\lambda_{l}$-dependence of $F_{G, \sigma}$ on each Landau singularity is also easily seen from the proof, and we have

Corollary 6.3. $F_{G, \sigma}(p ; \lambda)$ is continued as a meromorphic function of $\lambda \in C^{N}$, and on $\Lambda_{\left(G-I^{i}\right) / I^{u}, \tau_{I}^{u} \sigma^{u}}^{+}$it is holomorphic except for (possible) simple poles at

$$
\begin{array}{ll}
\sum_{l \in I} \lambda_{l}-\frac{1}{2} \nu b_{1}\left(G_{1}\right)=0,-1,-2, \cdots & \left(I \subset I^{u}\right), \\
\sum_{l^{\prime} \in I^{\prime}} \lambda_{l^{\prime}}-\frac{1}{2} \nu b_{1}\left(Q_{I^{\prime}}\right)=0,1,2, \cdots & \left(I^{\prime} \subset I^{i}\right) .
\end{array}
$$

Remark. This is not the best possible estimate for the poles in $\lambda$. Precise results are obtained by Speer [14].

In order to define $F_{G, \sigma}(p ; \lambda)$ at $\lambda_{l}=1\left(l \in I_{0}\right)$, we follow Speer's analytic renormalization procedure. Since application of an evaluator does not increase the singularity spectrum, (6.4) remains valid for renormalized Feynman integrals. We shall show below the equivalence of our $F_{G, \sigma}$ to Speer's generalized Feynman amplitude [13], which assures that this 
renormalization is also equivalent to that of $\mathrm{BPH}$. Let $(G, \sigma)$ be a massive graph. Define

$$
\begin{aligned}
& f_{G, \sigma}(p ; \lambda)=\gamma_{G}(\lambda) \sum_{\text {perm }}^{N !} \int_{0}^{1} \cdots \int_{0}^{1} \prod_{l=1}^{N} d \beta_{l} \cdot \delta\left(1-\sum_{l=1}^{N} \beta_{1} \cdots \beta_{l}\right) \\
& \text { - } \prod_{l=1}^{N} \beta_{l}{ }^{\lambda\left(G_{l}\right)-1} \widetilde{U}(\beta)^{-\nu / 2}(\widetilde{V}(p, \beta)-i 0)^{-\lambda(G)} \text {. }
\end{aligned}
$$

Here we have set

$$
\begin{gathered}
\gamma_{G}(\lambda)=i^{N}(2 \pi)^{\nu(N-n)} e^{-\pi i \lambda(G)}(-i)^{(\nu-1) b_{1}(G)} \pi^{\frac{1}{2} \nu b_{1}(G)} \Gamma(\lambda(G)) \prod_{l \in I_{0}} \Gamma\left(\lambda_{l}\right)^{-1}, \\
\lambda(G)=\sum_{l \in I_{0}} \lambda_{l}-\frac{1}{2} \nu b_{1}(G), \\
G_{l}=G_{\{1,2, \ldots, l\}},
\end{gathered}
$$

and the summation extends over all the possible orderings of the internal lines. The functions $\widetilde{U}(\beta)$ and $\widetilde{V}(p, \beta)$ are defined from the $U$-function and $V$-function (Nakanishi [8]) by the following formula:

$$
\begin{aligned}
& \left.U_{G}(\alpha)\right|_{\alpha_{l} \mapsto \beta_{1} \cdots \beta_{l}}=\prod_{l=1}^{N} \beta_{l}^{b_{l}\left(G_{l}\right)} \widetilde{U}(\beta), \\
& \widetilde{V}(p, \beta)=\left.\left(-U_{G}(\alpha)^{-1} \sum_{i \neq j} U_{G_{i j}}(\alpha) q_{i} q_{j}+\sum_{l=1}^{N} \alpha_{l} m_{l}{ }^{2}\right)\right|_{\alpha_{l} \mapsto \beta_{1} \cdots \beta_{l}}
\end{aligned}
$$

where $q_{j}=\sum_{i \in \sigma^{-1}(j)} p_{i}$ and $G_{i j}$ denotes the graph obtained from $G$ by identifying the two vertices $\{i, j\}$. Note that $\widetilde{U}(\beta)=1+O(\beta)$ when $\beta_{l} \rightarrow 0$ for $l \in I_{0}$, $l \neq 1$.

Theorem 6.4. For a massive graph $(G, \sigma)$ we have

$$
F_{G, \sigma}(p ; \lambda)=\delta\left(\sum_{i \in J_{0}} p_{i}\right) \cdot f_{G, \sigma}(p ; \lambda) \text {. }
$$

Proof. Since both hand sides are meromorphic with respect to $\lambda \in \mathbb{C}^{N}$, we may assume $\operatorname{Re} \lambda_{l} \gg 0$ without loss of generality. For $\varepsilon>0$, set

$$
\Phi_{G, \sigma}^{\varepsilon}(p, k ; \lambda)=i^{N}(2 \pi)^{\nu(n-N)} \prod_{j=1}^{n} \delta\left(q_{j}-\sum_{l \in I_{0}}[l: j] k_{l}\right) \prod_{l \in I_{0}}\left(k_{l}{ }^{2}-m_{l}{ }^{2}+i \varepsilon\left|k_{l}\right|^{2}\right)^{-\lambda_{l}} d k,
$$

where $\mid k_{1}^{2}=\sum_{\mu=0}^{\nu-1} k_{\mu}{ }^{2}$. In the same way as $\Phi_{G}, \Phi_{G}{ }^{\varepsilon}$ may be extended to to $\boldsymbol{R}^{\nu n_{0}} \times \widetilde{X}$ as a (holonomic) hyperfunction density. The proof goes in three 
steps.

Lemma 6.5. As a distribution on $\boldsymbol{R}^{\nu n}$ we have

$$
F_{G, \sigma}(p ; \lambda)=\lim _{\varepsilon \downarrow 0} \int_{\widetilde{X}} \Phi_{G, \sigma}^{\varepsilon}(p, k ; \lambda) .
$$

Proof. The integration being a continuous operation, it suffices to prove $\Phi_{G, \sigma}(p, k ; \lambda)=\lim _{\varepsilon \downarrow 0} \Phi_{G, \sigma}^{\varepsilon}(p, k ; \lambda)$ on $\boldsymbol{R}^{\nu n_{0}} \times \widetilde{X}$. In terms of local coordinates $\bar{\Phi}_{G, \sigma}^{\varepsilon}$ has an expression similar to $(6 \cdot 5)$ :

$$
\Phi_{G, \sigma}^{\varepsilon}=i^{N}(2 \pi)^{\nu(n-N)} \delta \widetilde{V} \prod_{\rho=1}^{r}\left|t_{\rho}^{u}\right|^{\lambda_{\rho}^{u}} \prod_{l \in I_{0}}\left(k_{l}{ }^{2}-s_{l}(t)^{2} m_{l}{ }^{2}+i \varepsilon\left|k_{l}\right|^{2}\right)^{-\lambda_{l}}\left|d k d t^{u}\right| .
$$

From (6.9) one may show that $\Phi_{G, \sigma}=\lim _{\varepsilon \downarrow 0} \Phi_{G, \sigma}^{\varepsilon}$. We leave the details to the reader.

\section{Lemma 6.6.}

$$
\int_{\widetilde{X}} \Phi_{G, \sigma}^{\varepsilon}(p, k ; \lambda)=\int_{\boldsymbol{R}^{\nu N}} \Phi_{G, \sigma}^{\varepsilon}(p, k ; \lambda) .
$$

Proof. Note that after eliminating the delta functions the right hand side reduces to an absolutely convergent integral for $\operatorname{Re} \lambda_{l} \gg 0$ apart from the overall delta function. Since $\operatorname{Re} \lambda_{\rho}^{u}>0$ in (6.9) we may regard $\Phi_{G, \sigma}^{\varepsilon}$ as a continuous function on $\widetilde{V}$, so it is easy to see that there is no contribution from $\widetilde{X}-X^{\prime}=\widetilde{S}_{I_{0}}$.

\section{Lemma 6.7 .}

$$
\begin{gathered}
\int_{R^{\nu N}} \Phi_{G, \sigma}^{\varepsilon}(p, k ; \lambda)=\left((1+i \varepsilon)(1-i \varepsilon)^{\nu-1}\right)^{-(1 / 2) b_{1}(G)} \gamma_{G}(\lambda) \cdot \delta\left(\sum_{i \in J_{0}} p_{i}\right) \\
\cdot \sum_{\text {perm }}^{N !} \int_{0}^{1} \cdots \int_{0}^{1} \prod_{l=1}^{N} d \beta_{l} \cdot \delta\left(1-\sum_{l=1}^{N} \beta_{1} \cdots \beta_{l}\right) \prod_{l=1}^{N} \beta_{l}^{\lambda\left(G_{l}\right)-1} \widetilde{U}(\beta)^{-\nu / 2} \\
\cdot\left(\widetilde{V}(p, \beta)-i \varepsilon \widetilde{V}_{\alpha}(p, \beta)_{E}\right)^{-\lambda(G)}
\end{gathered}
$$

where $\widetilde{V}_{\alpha}(p, \beta)_{E}=\left.U_{G}(\alpha)^{-1} \sum_{i \neq j} U_{G_{i j}}(\alpha)\left(q_{i} q_{j}\right)_{E}\right|_{\alpha_{l 1 \rightarrow \beta_{1} \cdots \beta_{l}}}$, and $\left(q q^{\prime}\right)_{E}=\sum_{\mu=0}^{\nu-1} q_{\mu} q_{\mu}{ }^{\prime}$ denotes the Euclidean inner product.

Proof. After applying the Feynman's parametric formula to the left 
hand side, we divide the domain $\left\{\alpha \in \mathbb{R}^{N} \mid \alpha_{l} \geqq 0, \sum_{l \in I_{0}} \alpha_{l}=1\right\}$ to $N$ ! sectors of the form $0 \leqq \alpha_{1} \leqq \alpha_{2} \leqq \cdots \leqq \alpha_{N} \leqq 1$, change the coordinate by $\alpha_{l}=\beta_{1} \cdots \beta_{l}$ and integrate over $k$. All the calculations can be carried out within the category of absolutely convergent integrals, and finally we obtain (6-10).

In $(6 \cdot 10) \widetilde{V}_{\alpha}(p, \beta)_{E}$ is a positive definite quadratic form of $q$, hence $\lim _{\varepsilon \rightarrow 0}\left(\widetilde{V}(p, \beta)-i \varepsilon \widetilde{V}_{\alpha}(p, \beta)_{E}\right)^{-\lambda(G)}=(\widetilde{V}(p, \beta)-i 0)^{-\lambda(G)}$. From Lemma 6.5, $\stackrel{\varepsilon \rightarrow 0}{\text { Lemma }} 6.6$ and Lemma 6.7 follows the theorem.

Corollary 6.8. By a suitable choice of an evaluator, the following condition is satisfied simultaneously for any massive graph $(G, \sigma)$ :

$$
\overline{f_{G, \sigma}(p)}=(-)^{n(G)-b_{0}(G)} f_{G, \sigma}(p) \text { in a neighbourhood of } p=0 \text {. }
$$

In fact, from (6.8) one easily checks that the standard choice will do.

$$
W_{N}(f)=\frac{1}{N !} \sum_{\sigma \in \mathfrak{S}_{N}} \prod_{l \in I_{0}} \oint_{C_{\sigma(l)}} \frac{d \lambda_{l}}{2 \pi i} \frac{f(\lambda)}{\left(\lambda_{1}-1\right) \cdots\left(\lambda_{N}-1\right)} .
$$

Next we "quantize" the contact transformation of $\S 2$. In what follows $G$ will denote an external graph. The holonlmic system satisfied by the generalized Feynman integral $F_{G, \sigma}(p ; \lambda)$ on its leading Landau variety $\Lambda_{G}{ }^{+}$is given as follows (Sato [10]):

$$
\left(p_{j}-\sum_{l \in I_{0}}[l: j] \Phi_{l}^{*}\left(-i D_{p}\right)\right) F_{G}(p ; \lambda)=0 \quad(j=1, \cdots, n),
$$

where $\Phi_{l}^{*}(x)=\frac{m_{l} \eta_{l}(x)}{\sqrt{\eta_{l}(x)^{2}}} L_{\frac{\nu}{2}-\lambda_{l}}\left(m_{l} \sqrt{\eta_{l}(x)^{2}}\right)$,

(the branch of square root is taken so that $\sqrt{z}=1$ at $z=1$ ),

$$
\begin{aligned}
& L_{\frac{\nu}{2}-\lambda_{l}}(t)=i \frac{d}{d t} \log F_{\frac{\nu}{2}-\lambda_{l}}(t), \\
& F_{\frac{\nu}{2}-\lambda_{l}}(t)=e^{-i t} t^{-\frac{\nu-\lambda_{l}}{2}}{ }_{2} F_{0}\left(\frac{\nu+1}{2}-\lambda_{l}, \frac{1-\nu}{2}+\lambda_{l} ; \frac{1}{2 i t}\right) .
\end{aligned}
$$

Its order and principal symbol are

$$
\text { order }=\frac{1}{2}\left(\sum_{l \in I_{0}} \lambda_{l}+\nu \chi(G)\right) \text {, }
$$




$$
\text { principal symbol }=\prod_{l \in I_{0}}\left(m_{l} \sqrt{\eta_{l}(x)^{2}-i 0}\right)^{-\frac{\nu-\lambda_{l}}{2}} \sqrt{d x} .
$$

With the aid of this system, we define

$$
\begin{aligned}
& \widetilde{p}_{j}=p_{j}-\sum_{l \in I_{0}-I}[l: j] \Phi_{l} *\left(-i D_{p}\right), \\
& D_{\widetilde{p}_{j}}=D_{p_{j}} .
\end{aligned}
$$

This is actually a "quantized" contact transformation (QCT) corresponding to $(2 \cdot 1)$ of $\S 2$, and realized by an integral transformation with the kernel function

$$
K(p, \widetilde{p})=F_{G_{I_{0}-I}}(p-\widetilde{p}) .
$$

The transform of $F_{G}$ by $(6 \cdot 12)$ is $F_{G_{I}}$, in other words

$$
F_{G}(p ; \lambda)=\int F_{G_{I_{0}-I}}(p-p ; \lambda) F_{G_{I}}(\widetilde{p} ; \lambda) d \widetilde{p}
$$

holds on $\bigcup_{I_{1} \subset I} \Lambda_{G / I_{1}, \tau_{I_{1}}}^{+}$. To see this, note that on $\Lambda_{G / I_{1}, \tau_{I_{1}}}^{+}\left(I_{1} \subset I\right)$ there are no contributions from $\widetilde{S}_{I_{0}-I}$, so that in the definition of $F_{G}(p ; \lambda)$ we may restrict the region of integration to $\widetilde{X}_{G}-\widetilde{S}_{I_{0}-I}=\left(\boldsymbol{R}^{\nu}\right)^{I_{0}-I} \times \widetilde{X}_{G_{I}}$. It is now easy to see that on $\widetilde{X}_{G}-\widetilde{S}_{I_{0}-I}$

$$
\Phi_{G}(p, k ; \lambda)=\int \Phi_{G_{I_{0}-I}}(p-\widetilde{p}, k ; \lambda) \Phi_{G_{I}}(\widetilde{p}, k ; \lambda) d \widetilde{p}
$$

holds. Integration with respect to $k$ in $\widetilde{X}_{G}-\widetilde{S}_{I_{0}-I}$ gives $(6 \cdot 13)$.

So we have the correspondences just as in $(2 \cdot 2)$ :

$$
\begin{aligned}
& F_{G} \leftrightarrow F_{G_{I}}, \\
& F_{G / I, \tau_{I}} \leftrightarrow F_{G_{I^{\prime} /, \tau_{I}}}=\prod_{i=1}^{r} \delta\left(\sum_{j \in v\left(G_{I^{i}}\right)} p_{j}\right),
\end{aligned}
$$

where $G_{I}^{i}(1 \leqq i \leqq r)$ denotes the connected components of $G_{I}$, and $v\left(G_{I}{ }^{i}\right)$ the set of vertices of $G_{I}^{i}$. On the "zero section" $\Lambda_{G_{I} / I, \tau_{I}}^{+}, F_{G_{I}}$ is of the form $f_{G_{I}}(\widetilde{p} ; \lambda) \prod_{i=1}^{r}\left(\sum_{j \in v\left(G_{I^{i}}\right)} p_{j}\right)$ with an analytic function $f_{G_{I}}(p ; \lambda)$ (the generalized Feynman amplitude). By (6.12) this multiplication operator $f_{G_{I}}(p ; \lambda)$ is transformed into a general 0 -th order microdifferential operator.

Theorem 6.9. On $\Lambda_{G / I, \tau_{I}}^{+}$the following holds:

$$
F_{G}(p ; \lambda)=f_{G_{I}}\left(p_{j}-\sum_{l \in I_{0}-I}[l: j] \Phi_{l}^{*}\left(-i D_{p}\right) ; \lambda\right) \cdot F_{G / I, \tau_{I}}(p ; \lambda)
$$


This is a microlocal version of the local decomposition theorem disscussed in Speer-Westwater [16]. Note that the right hand side is well defined since the 0 -th order operators $\widetilde{p}_{j}=p_{j}-\sum_{l \in I_{0}-I}[l: j] \Phi_{l}{ }^{*}\left(-i D_{p}\right)(j=1, \cdots, n)$ commute each other. Extension of above results to the variable mass case is straightforward. We merely list up the results:

(holonomic system)

$$
\begin{aligned}
& \left(p_{j}-\sum_{l \in I_{0}-I}[l: j] D_{\mu_{l}}^{-1} \eta_{l}\left(D_{p}\right)\right) F_{G}(p, \mu ; \lambda)=0, \\
& \left(\mu_{l}+\frac{1}{2} D_{\mu_{l}}^{-1} \eta_{l}\left(D_{p}\right)^{2}+\left(\lambda_{l}-\frac{\nu}{2}-1\right) D_{\mu_{l}}^{-1}\right) F_{G}(p, \mu ; \lambda)=0, \\
& \eta_{l}\left(D_{p}\right)=\sum_{j=1}^{n}[l: j] D_{p_{j}} .
\end{aligned}
$$

$(\mathrm{QCT})$

$$
\begin{array}{ll}
\widetilde{p}_{j}=p_{j}-\sum_{l \in I_{0}-I}[l: j] D_{\mu_{l}}^{-1} \eta_{l}\left(D_{p}\right), \\
\widetilde{\mu}_{l}= \begin{cases}\mu_{l}+\frac{1}{2} D_{\mu_{l}}^{-2} \eta_{l}\left(D_{p}\right)^{2}+\left(\lambda_{l}-\frac{\nu}{2}-1\right) D_{\mu_{l}}^{-1} & \left(\text { if } l \in I_{0}-I\right), \\
\mu_{l} & \text { (if } l \in I),\end{cases} \\
D_{\tilde{p}_{j}}=D_{p_{j}}, D_{\tilde{\mu}_{l}}=D_{\mu_{l}} .
\end{array}
$$

(kernel function)

$$
\begin{aligned}
& K(p, \widetilde{p}, \mu, \widetilde{\mu})=F_{G_{I_{0}-I}}\left(p-\widetilde{p}, \mu_{I_{0}-I}-\widetilde{\mu}_{I_{0}-I}\right) \delta\left(\mu_{I}-\widetilde{\mu}_{I}\right), \\
& \mu_{I}=\left(\mu_{\imath}\right)_{l \in I} .
\end{aligned}
$$

(correspondence)

$$
\begin{aligned}
& \Lambda_{G}^{+} \leftrightarrow \Lambda_{G_{I}}^{+} \times\left\{\text {conormal bundle of } \widetilde{\mu}_{I_{0}-I}=0\right\}, \\
& F_{G}(p, \mu ; \lambda) \leftrightarrow F_{G_{I}}\left(\widetilde{p}, \widetilde{\mu}_{I} ; \lambda\right) \delta\left(\widetilde{\mu}_{I_{0}-I}\right) .
\end{aligned}
$$

Theorem 6.9'. On $\Lambda_{G / I, \tau_{I}}^{+}$,

$$
\begin{aligned}
F_{G}(p, \mu ; \lambda)= & f_{G_{I}}\left(p_{j}-\sum_{l \in I_{0}-I}[l: j] D_{\mu_{l}}^{-1} \eta_{l}\left(D_{p}\right), \mu_{I} ; \lambda\right) \\
& \cdot F_{G / I, \tau_{I}}\left(p, \mu_{I_{0}-I} ; \lambda\right) .
\end{aligned}
$$

If $L_{G / I, \tau_{I}}^{+}$is a hypersurface (i.e. $\operatorname{codim} L_{G / I, \tau_{I}}^{+}=\nu+1$ ), we can replace 
the operator $f_{G_{I}}(\widetilde{p}, \mu ; \lambda)$ by a multiplication of an analytic function (cf. Kashiwara [6]).

Theorem 6.10. (Speer-Westwater [15]) If G/I is primary, we have near a generic point of $L_{G / I, \tau_{I}}^{+}$the decomposition

$$
F_{G}(p, \mu ; \lambda)=A(p, \mu ; \lambda) F_{G / I, \tau_{I}}\left(p, \mu_{I_{0}-I} ; \lambda\right)+B(p, \mu ; \lambda),
$$

where $A(p, \mu ; \lambda), B(p, \mu ; \lambda)$ are analytic functions such that

$$
\left.A(p, \mu ; \lambda)\right|_{L_{G / I, \tau_{I}}^{+}}=f_{G_{I}}\left(p_{j}-\sum_{l \in I_{0}-I}[l: j] k_{l}(p, u), \mu_{I} ; \lambda\right)
$$

holds. Here $k_{l}(p, \mu)$ is the rational function defined in Proposition 4.10.

\section{$\S$ 7. The Generalized Unitarity Relations}

As an application of QCT in the previous section, we shall prove a thecrem which provides a consistency check of the generalized unitarity relations for time ordered Green's functions (Nishijima[9]) and perturbation theory.

Let $G$ be a massive connected external graph. Given a division $J_{0}$ $=J^{+}\left\llcorner J^{-}\right.$of the set of vertices, we define

$$
\begin{aligned}
F_{J^{+J}-}(p)= & \int \cdots \int_{R^{\nu}(\# S)} F_{G^{+}}\left(p^{+}\right) \prod_{l \in S} \theta\left([S: l] k_{l 0}\right) 2 \pi \delta\left(k_{l}{ }^{2}-m_{l}{ }^{2}\right) \\
& \times \overline{F_{G^{-}}\left(p^{-}\right)} \prod_{l \in S} \frac{d k_{l}}{(2 \pi)^{\nu}},
\end{aligned}
$$

with $p_{j}{ }^{ \pm}= \pm\left(p_{j}-\sum_{l \in S}[l: j] k_{l}\right)$. Here $G^{ \pm}$denotes the full subgraph corresponding to $J^{ \pm}, F_{G^{ \pm}}$the renormalized Feynman integral and $S$ the set of lines which do not belong to $G^{ \pm}{ }^{\dagger}$ ) The orientation of $S$ is chosen arbitrarily (Figure 7.1).

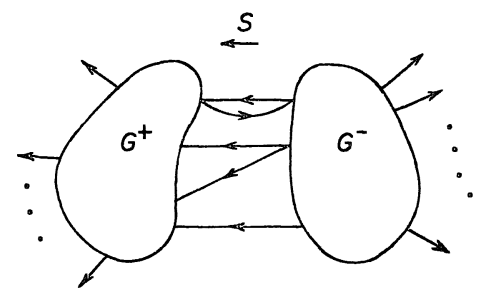

Fig. (7.1)

†) $\# S$ denotes the number of elements in the set $S$. 
Since for any $p$ the fibre of integration is contained in the compact set $\left\{k \in \mathbb{R}^{\nu \# S} \mid \sum_{l \in S}[S: l] k_{l}=\sum_{j \in J^{+}} p_{j},[S: l] k_{l 0}>0, k_{l}{ }^{2}=m_{l}{ }^{2}(l \in S)\right\},(7 \cdot 1)$ is always well defined.

Theorem 7.1. If the analytic renormalization is performed so that (6.11) is satisfied for any $G$, we have

$$
\sum_{J_{0}=J^{+}\left\lfloor J^{-}\right.}(-)^{\# J^{+}} F_{J^{+} J^{-}}(p)=0,
$$

where the summation extends over all the divisions of $J_{0}$.

Remark. $(7 \cdot 2)$ is also valid for non external $(G, \sigma)$ provided the substitution $\left.F_{J^{+J}-}(q)\right|_{q_{j} \rightarrow \underset{i \in \sigma^{-1}(j)}{\Sigma} p_{i}}$ is well defined.

Proof. If we denote the left hand side of $(7 \cdot 2)$ by $\mathscr{F}_{G}, S S \mathscr{F}_{G}$ is contained in the union of various mixed- $\alpha$ Landau singularities. We show inductively from the leading singularities that $\mathscr{F}_{G}=0$ on each of them.

Consider first a connected component $\Lambda$ of a leading singularity. We represent it by a graph as in Figure 7.2:


Fig. (7.2)

For example, the graph of Figure 7.2 represents the component $\{(p ; x)$ $\in T^{*} \boldsymbol{R}^{3 \nu} \mid \exists k_{l} \in \boldsymbol{R}^{\nu},{ }^{\exists} \alpha_{l} \in \boldsymbol{R}(l=1,2,3)$ such that (I) $p_{1}+k_{1}+k_{3}=0, p_{2}-k_{1}$ $-k_{2}=0, \quad p_{3}+k_{2}-k_{3}=0$, (II) $\alpha_{1} k_{1}=x_{2}-x_{1}, \quad \alpha_{1}<0, \quad \alpha_{2} k_{2}=x_{2}-x_{3}, \quad \alpha_{2}>0$, $\alpha_{3} k_{3}=x_{3}-x_{1}, \quad \alpha_{3}>0$, (III) $\left.k_{l}{ }^{2}=m_{l}{ }^{2}(l=1,2,3), \quad k_{10}>0, \quad k_{20}>0, \quad k_{30}<0\right\}$. There is at least one vertex $j_{0}$ to which only the dotted lines are incident. For, if not, we could find a circuit of Figure 7.3. Then the circuit condition $\sum[C: l] \alpha_{l} k_{l}=0$ would be incompatible with the sign of $\alpha_{l} k_{l 0}$. 


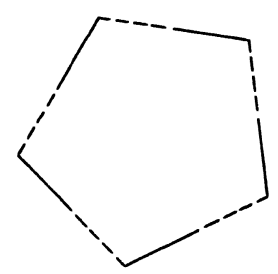

Fig. (7.3)

Take a term $F_{J^{+} J^{-}}$in $\mathscr{F}_{G}$ and suppose, for instance, $j_{0} \in J^{+}$. If we rewrite it as a sum of $2^{N}$ terms using

$$
\begin{aligned}
& \frac{i}{k^{2}-m^{2} \pm i 0}=\theta\left(k_{0}\right) \frac{i}{k^{2}-m^{2} \pm i 0}+\theta\left(-k_{0}\right) \frac{i}{k^{2}-m^{2} \pm i 0} \\
& \theta\left( \pm k_{0}\right) 2 \pi \delta\left(k^{2}-m^{2}\right)=\theta\left( \pm k_{0}\right)\left(\frac{i}{k^{2}-m^{2}+i 0}-\frac{i}{k^{2}-m^{2}-i 0}\right)
\end{aligned}
$$

we observe that only one term survives on $\Lambda$, and that this term cancels out with the corresponding one in $F_{J^{+}-\left\{j_{0}\right\}, J-\sqcup\left\{j_{0}\right\}}$ (Figure 7.4).
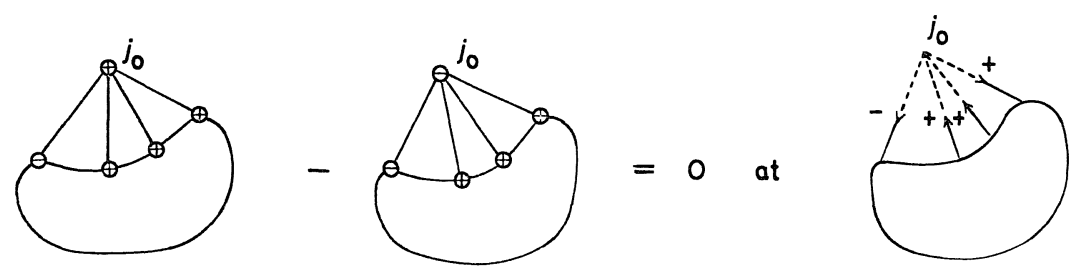

Fig. (7.4)

Next we assume $\mathscr{F}_{G}=0$ on any of the connected components of $\Lambda_{G / I^{\prime}, \tau_{I^{\prime}}}^{\text {mixed }}$ $\left(I^{\prime} \varsubsetneqq I\right)$, and prove that it also vanishes on $\Lambda_{G / I, \tau_{\tau^{*}}}^{\text {mixed }}$ Applying QCT (6.12), $\mathscr{F}_{G}$ is transformed to the form $g(\widetilde{p}) \times \prod_{i=1}^{r} \delta\left(\sum_{j \in v\left(G_{I} I^{i}\right.} \widetilde{p}_{j}\right)$, where by the induction assumption $g(\tilde{p})$ is an analytic function with no singularities. Note that if $S \cap G_{I}^{i} \neq \phi$ for some $i$, then the transform of $F_{J^{+} J^{-}}$does not have its support in an neighbourhood of $\widetilde{p}=0$. If $S \cap G_{I}{ }^{i}=\phi(1 \leqq i \leqq \mathrm{r})$, we may apply the local decomposition (Theorem 6.9) to $F_{J^{+} J-\text {. Then }}$ condition (6.11) assures the pairwise cancellation in a neighbourhood of $\widetilde{p}=0$ just as in Figure 7.4 , where the signed vertices are replaced by the signed bubbles representing the contracted subgraphs $G_{I}{ }^{i}$. Hence by the unique continuation property of analytic functions we conclude $g(\tilde{p})=0$ on any of the connected components of $\Lambda_{G / I, \tau_{I}}^{\text {mixed }}$. This completes 
the proof of the theorem.

The generalized unitarity relations, regarded as an identity of formal power series (the perturbation expansion of $\tau$-functions), are equivalent to a set of equations which are linear combinations of $(7 \cdot 2)$. Hence Theorem 7.1 provides a perturbation theoretical "proof" of unitarity. Our discussion is only formal, though, since we have not taken into account the delicate problem concerning renormalization.

\section{References}

[1] Boyling, J. B., A homological approach to parametric Feynman integrals, Nuovo Cimento, 53A (1968), 591-614.

[2] Chandler, C., Some physical region mass shell properties of renormalized Feynman integrals, Comm. Math. Phys., 19 (1970), 169-188.

[3] Chandler, C. and Stapp, H. P., Macroscopic causality conditions and properties of scattering amplitudes, J. Math. Phys., 90 (1969), 826-859.

[4] Fotiadi, D., Désingularisation et graphs de Feynman, Séminaire Collège de France (1969).

[5] Iagolnitzer, D. and Stapp, H. P., Macroscopic causality and physical region analyticity in $S$-matrix theory, Comm. Math. Phys. 14 (1969), 15-55.

[6] Kashiwara, M., Microlocal calculus, Lecture Notes in Phys. 39, Springer, BerlinHeidelberg-New York (1975), 30-37.

[7] Kashiwara, M. and Kawai, T., this proceedings.

[8] Nakanishi, N., Graph theory and Feynman integrals, Gordon and Breach (1971).

[9] Nishijima, K., Asymptotic conditions and perturbation theory, Phys. Rev., 119 (1960), 485-498.

[10] Sato, M., Recent development in hyperfunction theory and its application to physics, Lecture Notes in Phys. 39, Springer (1975), 13-29.

[11] Sato, M., Kawai, T. and Kashiwara, M., Microfunctions and pseudo-differential equations, Lecture Notes in Math., 287, Springer, (1973), 265-529.

[12] Sato, M., Miwa, T. and Jimbo, M., Dimension formula for the Landau singularity, Sûrikaisekikenkyusho Kôkyûroku, 266, RIMS, Kyoto Univ. (1976), 91-107.

[13] Speer, E. R., Generalized Feynman Amplitudes, Princeton University Press, Princeton, 1969.

[14] - Ultraviolet and infrared singularity structure of generic Feynman amplitudes, Ann. Inst. H. Poincaré, 23 (1975), 1-21.

[15] Speer, E. R, and Westwater, M. J., Generic Feynman Amplitudes, Ann. Inst. H. Poincaré, 14 (1971), 1-55.

Note added in proof. Since the fact that $\Phi_{G, \sigma}^{\varepsilon} \rightarrow \Phi_{G, \sigma}$ in the proof of Lemma 6.5 does not seem quite obvious, we shall give a proof to it in what follows. One of the authors is indebted to Professor Kawai for his kind remark. 


\section{Errata}

A Correction to: "Holonomy Structure of Landau Singularities and Feynman integrals" (Vol. 12, suppl. 1977, 387-438)

by

\section{Michio Jiмво}

The following lemma should be inserted between Lemma 6.5 and Lemma 6.6.

Lemma. $\quad \lim _{\diamond \downarrow 0} \Phi_{G, \sigma}^{\mathrm{e}}=\Phi_{G, \sigma}$

Proof. Denote by $\widetilde{V}^{c}$ a complexification of the manifold $\widetilde{V}$, and set

$$
f_{\varepsilon}=\prod_{\rho=1}^{r}\left( \pm t_{\rho}^{u}\right)^{\lambda_{\rho}^{u}} \prod_{l \in I_{o}}\left(k_{l}{ }^{2}-s_{l}(t)^{2} m_{l}{ }^{2}+i \varepsilon \sum_{\mu=0}^{\nu-1} k_{l_{\mu}}^{2}\right)^{-\lambda_{l}},
$$

where $\varepsilon \geqq 0$ and $t_{\rho}^{u}, k_{l}$ stand for polynomial functions on $\widetilde{V}^{c}$ (see Proposition 6.1 and the Remark following it). For a point $\widetilde{P}_{0} \in \widetilde{V}$ we set $I=\left\{l \in I_{0} \mid k_{l}{ }^{2}-s_{l}(t)^{2} m_{l}{ }^{2}=0\right.$ at $\left.\widetilde{P}_{0}\right\}$. Since the product (6.5) is well defined, we may find a complex neighborhood $\Omega$ of $\widetilde{P}_{0}$ and an open convex cone $\Gamma$ so that for any $\omega \Subset U=\Omega \cap \widetilde{V}$ and $\Gamma^{\prime} \Subset \Gamma$ there exists $C>0$ such that

$$
\begin{aligned}
& \operatorname{Im}\left( \pm t_{\rho}^{u}\right)>C|\operatorname{Im} z|>0, \\
& \operatorname{Im}\left(k_{l}{ }^{2}-s_{l}(t)^{2} m_{l}{ }^{2}\right)>C|\operatorname{Im} z|>0 \quad\left(z \in \Omega \cap\left(\omega+i \Gamma^{\prime}\right), l \in I\right)
\end{aligned}
$$

hold. Here $z$ denotes a local coordinate of $\widetilde{V}^{c}$. For any $l \in I$, noting $k_{l} \neq 0$ at $\widetilde{P}_{0}$ we see $\left|\operatorname{Re} k_{l}\right|$ is bounded from below, hence replacing $\Omega$ by $\Omega \cap\left\{\left.z|| \operatorname{Re} k_{l}\right|^{2}>\left|\operatorname{Im} k_{l}\right|^{2}(l \in I)\right\} \quad$ we may assume $\operatorname{Im}\left(i \sum_{\mu=0}^{\nu-1} k_{l_{\mu}}^{2}\right)>0$ on $\Omega$. Hence $f_{\varepsilon}(\varepsilon \geqq 0)$ is holomorphic in $\Omega \cap(U+i \Gamma)$, and we have

$$
\begin{aligned}
\left|f_{\varepsilon}(z)-f_{0}(z)\right| & =\prod_{\rho=1}^{r}\left| \pm t_{\rho}^{u}\right| \lambda_{\rho}^{u}\left|\int_{0}^{\varepsilon} d \theta \frac{\partial}{\partial \theta} \prod_{l \in I_{0}}\left(k_{l^{2}}{ }^{2}-s_{l}(t)^{2} m_{l}{ }^{2}+i \theta \sum_{\mu=0}^{\nu-1} k_{l_{\mu}}^{2}\right)^{-\lambda_{l}}\right| \\
& \leqq \varepsilon C^{\prime} \sum_{l \in I_{0}} \prod_{l^{\prime} \in I_{0}}\left|\operatorname{Im}\left(k_{l^{\prime}}^{2}-s_{l^{\prime}}(t)^{2} m_{l^{\prime}}{ }^{2}\right)\right|^{-\left(\lambda l^{\prime}+\delta l^{\prime}\right)}
\end{aligned}
$$

* Research Institude for Mathematical Sciences, Kyoto University, Kyoto 606, Japan. 


$$
\leqq \varepsilon C^{\prime \prime}|\operatorname{Im} z|^{-L}
$$

for some $C^{\prime \prime}, L>0$ independent of $\varepsilon$. Similarly we have $\left|f_{\varepsilon}(z)\right|$ $\leqq C^{\prime \prime}|\operatorname{Im} z|^{-L}$. By the same argument as in Nishiwada [1], it then follows that $f_{\varepsilon}(x+i 0 \Gamma)$ exists in $\mathscr{D}(U)$ and $\lim _{\varepsilon \rightarrow 0} f_{\varepsilon}(x+i 0 \Gamma)=f_{0}(x+i 0 \Gamma)$. Writing $\Phi_{G, \sigma}^{\varepsilon}$ and $\Phi_{G, \sigma}$ as linear combinations of $\delta_{\widetilde{v}} f_{\varepsilon}(x+i 0 \Gamma)$, we have the lemma.

\section{Reference}

[1] Nishiwada, K., On local characterization of wave front sets in terms of boundary values of holomorphic functions, to appear in Publ. RIMS. 
